\title{
Wireless Sensing Based on RFID and Capacitive Technologies for Safety in Marble Industry Process Control
}

\author{
Fabrizio Iacopetti, Sergio Saponara, Luca Fanucci, and Bruno Neri \\ Department of Information Engineering, University of Pisa, Via Caruso 16, 56122 Pisa, Italy \\ Correspondence should be addressed to Fabrizio Iacopetti; fabrizio.iacopetti@iet.unipi.it
}

Received 29 July 2012; Accepted 19 October 2012

Academic Editor: Agusti Solanas

Copyright (C) 2013 Fabrizio Iacopetti et al. This is an open access article distributed under the Creative Commons Attribution License, which permits unrestricted use, distribution, and reproduction in any medium, provided the original work is properly cited.

\begin{abstract}
This paper presents wireless sensing systems to increase safety and robustness in industrial process control, particularly in industrial machines for marble slab working. The process is performed by abrasive or cutting heads activated independently by the machine controller when the slab, transported on a conveyer belt, is under them. Current slab detection systems are based on electromechanical or optical devices at the machine entrance stage, suffering from deterioration and from the harsh environment. Slab displacement or break inside the machine due to the working stress may result in safety issues and damages to the conveyer belt due to incorrect driving of the working tools. The experimented contactless sensing techniques are based on four RFID and two capacitive sensing technologies and on customized hardware/software. The proposed solutions aim at overcoming some limitations of current state-of-the-art detection systems, allowing for reliable slab detection, outside and/or inside the machine, while maintaining low complexity and at the same time robustness to industrial harsh conditions. The proposed sensing devices may implement a wireless or wired sensor network feeding detection data to the machine controller. Data integrity check and process control algorithms have to be implemented for the safety and reliability of the overall industrial process.
\end{abstract}

\section{Introduction}

The transformation of stone blocks coming from quarries into finished products, for example, tiles, sculptures, building materials, stone powder, and so forth, is performed through several different industrial processes. In the case of slabshaped products (e.g., tiles), marble blocks coming from quarries are firstly sawn by a gangsaw [1] into marble slabs with a resulting irregular contour and with rough surfaces. Slabs are afterwards polished in a polishing machine [2], cut into smaller and regular slabs by means of cutting machines, and finally become end products. The process control of marble slab working is nowadays mainly based on a feedforward control scheme: the marble slab is transported inside the machine by a conveyer belt; at the entrance of the machine, contact or optical sensing technologies are used to derive information on the presence and shape of the slab which are then used by the machine controlling system to drive the working heads on the slab when it is passing under them. Due to unforeseen events that may occur to the slab inside the machine, mainly slab displacement and cracks, the controlling system may drive the working heads on the base of not up-to-date or wrong information on the shape and position of the slab. This leads to damages for the machine, in particular for the conveyer belt, with resulting costs due to the need of replacing damaged parts and above all to the machine stop. The detection of slab cracks and of other working problems is nowadays still demanded to operators supervising the machine during the course of the working, who stop the machine in case of suspected or occurred problems. This approach may result in late intervention and in consequent machine damages but also may increase the safety risks for the workers. Moreover, the currently industrially used slab sensing technologies suffer from some issues like deterioration and performance problem in the dirty and wet working environment (mud, water, and stone residuals).

For the above-mentioned reasons, a feedback control scheme on the slab position inside the machine would turn into an improvement for the reliability and safety of the industrial process control. 
This paper deals in particular with the use of contactless sensing technologies, specifically based on RFID (radio frequency identification) and capacitive techniques, and to a multipoint wireless sensing data generation approach to improve the reliability and safety in industrial machines for marble slabs polishing. The proposed approach can be generally applied to other machines for stone slab working (cutting, waxing, etc.). The use of wireless technologies in industrial applications is an interesting and emerging trend, aiming at reducing cabling and installation complexity and costs and at avoiding the danger of cables and connectors failure especially on moving parts of industrial machines. Several works have been proposed in the literature [3] mainly addressing wireless systems for industrial communication or, in the case of RFID techniques, for positioning and logistics. On the contrary this work exploits RFID wireless technologies both for contactless multipoint sensing and wireless data communication; the work proposes and examines also the use of multipoint capacitive sensing techniques. For both RFID- and capacitive-based sensing applications, hardware and software components have been developed or COTS (commercial off the shelf) devices have been characterized and proposed for an integration within the machine process control architecture. This approach is supported by experimental campaigns on sensor performance using both sensing wireless technologies (RFID and capacitive) and considering working environments representative of those found in real industrial applications.

After this introduction this work briefly reviews in Section 2 the working principle of industrial marble machines and the state-of-the-art solutions based on optical and mechanical sensors and their limits. Section 3 introduces 4 RFID systems analyzed and tested in the present work, each one based on a different RFID technology, to try overcoming through contactless and wireless sensing the limits of the state-of-the-art process control applications. Then, in Section 4 to 7 , the work presents the applications and results concerning the test of the 4 mentioned RFID technologies applied to the case of slab detection in conditions representative of a real industrial working scenario. Section 8 presents the basic principle for capacitive sensing (capacitive sensor and the relevant front-end acquisition circuitry), while Section 9 presents two different types of capacitive sensors that have been designed, implemented, and tested in a test setup representative of real industrial working conditions. A comparison among the different analyzed contactless and wireless sensing solutions, based on RFID and capacitive technologies, is reported in Section 10. Conclusions are drawn in Section 11.

\section{Process Control and Sensors in Industrial Marble Machines}

Figure 1 shows the schematic diagram of a typical industrial marble machine for slab working. Marble machines are typically made up of consecutive working heads (up to a few tens) under which the marble slab, initially brought to the machine by means of a roller system, is transported by a plastic conveyer belt [2]. A marble slab is typically sized $2 \mathrm{~m} \times 3 \mathrm{~m}$ and has a thickness of some $\mathrm{cm}$. The typical conveyer belt speed amounts to a few $\mathrm{cm} / \mathrm{s}$. As an example, the polishing machine in [2] has a total length of $13.5 \mathrm{~m}$ and up to 18 abrasive heads.

In Figure 2 the schematic illustration of a section constituted by the different materials/parts inside the machine is reported.

The working environment inside a marble machine is dirty, due to mud, marble, and abrasive residuals, and wet, due to a water level of few $\mathrm{cm}$ needed for heads cooling, elimination of residuals, and easing of the conveyer belt sliding on the machine metallic plane. These mentioned issues add up to the other classic problems of wireless systems in industrial scenarios, such as electromagnetic signal attenuation, multipath, and interference from other electromagnetic sources like electrical motors.

As mentioned in Section 1, the process control for marble slab working is currently based on the following scheme: mechanical or optical sensors, arranged in a linear array at the entrance of the machine, are sampled at regular intervals (i.e., each second) to detect the presence of the marble slab on the conveyer belt at the entrance of the industrial machine (profile reader in Figure 1). Such approach results in a spatial sampling of the slab area, with sample step of a few $\mathrm{cm}$, which is used by the PLC (programmable logic controller) controlling the entire machine, including the conveyer belt speed, as the reference time to drive down each cutting/abrasive head when the marble slab is available and to drive up the head in the initial position when the slab is overpassing the working tool. The above-mentioned feed-forward control rule is based on the assumptions that the position and speed of the conveyer belt, the position of the marble slab on the belt, and therefore the relative positions of the heads are constantly known. If one of such assumptions is not verified, in particular the position of the slab on the belt, then the marble slab may not be present when the head is driven down, so that the latter reaches and damages the conveyer belt which must be repaired or replaced causing a long stop of the machine and of the industrial production. During the long travel inside the machine, the slab might indeed move on the conveyer belt due to the working forces or due to breaks under the working mechanical stress. Hence a feedback detection signal should be provided to the heads control system about the real presence of the marble slab under the head inside the machine.

In addition to the missing feedback to the heads control system inside the machine, the state-of-the-art sensors for slab detection outside the machine have problems still to overcome: mechanical sensors suffer from deterioration due to the continuous contact with the slab, while optical sensors [4] need frequent cleaning and recalibration due to the dirty working environment; see Figure 3.

Due to the wet and dirty working conditions and to the nonhomogeneous and nonconstant environmental physical properties, also other contactless sensors proposed or potentially suitable for marble machines, based on LASER or vision systems or ultrasonic waves [5-8], turned out not to be suitable for successful industrial applications.

Capacitive sensors for marble [9] and more complex ultrasound- or georadar-based systems $[6,8,10]$ have been studied in the literature. However, their target is the 


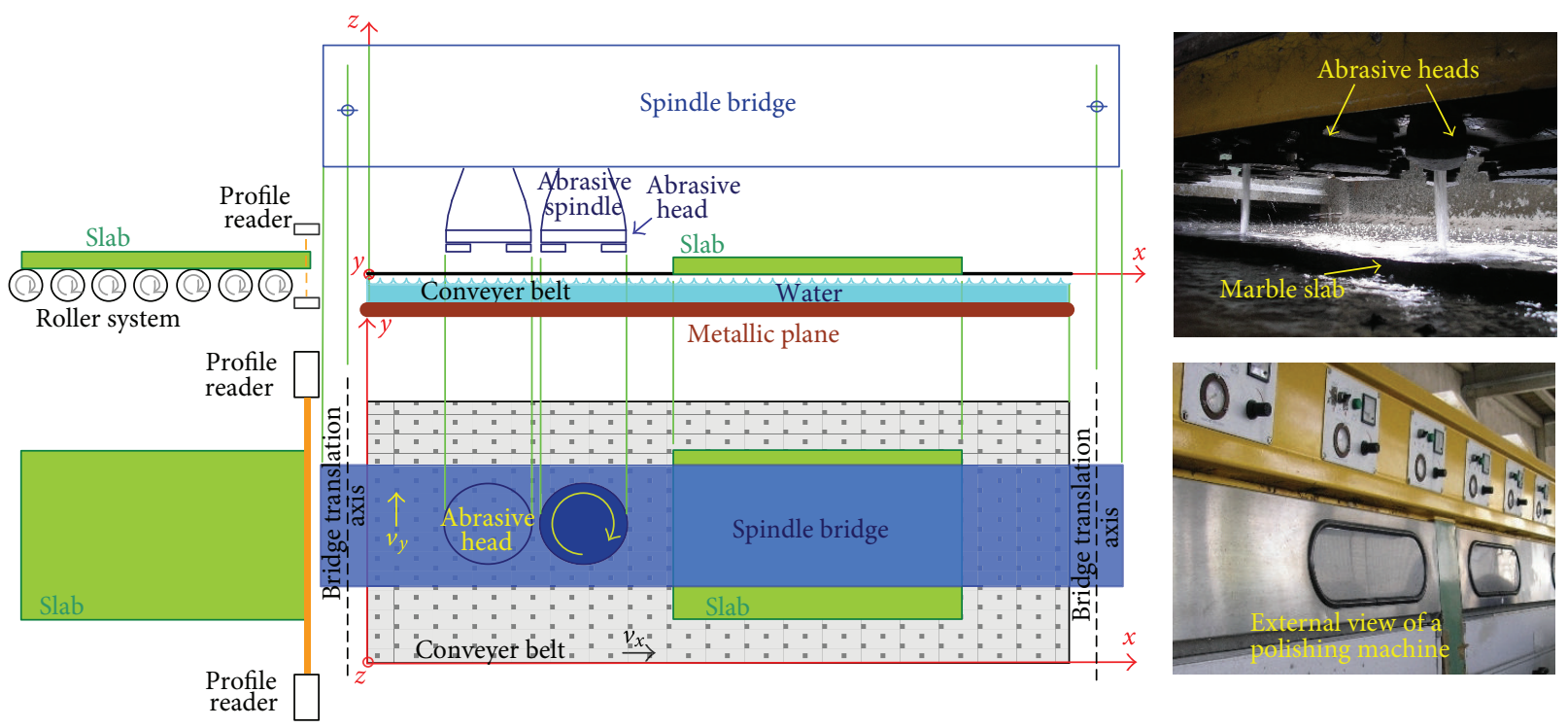

FIGURE 1: Schematic diagram of a marble machine and a snapshot of the abrasive heads over a marble slab.

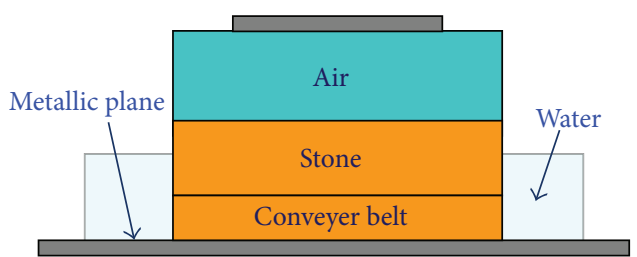

FIGURE 2: Schematic representation of a section of the different materials/parts inside the machine.

fine-grain analysis of the porosity and defects of stone materials (e.g., measuring the dielectric permittivity variations) in a controlled working environment (dry, clean, and with still stone samples) rather than the real-time detection of the presence of a marble slab during the working process inside an industrial machine.

In [11] we have presented preliminary results from experimental tests exploiting capacitive sensing for the detection of the marble slab at the entrance and inside the marble machine, which are further illustrated in Sections 9.1 and 9.2.

Proximity capacitive sensors have also been proposed by semiconductor industry in [12] but targeting small distances mainly for touch sensing applications.

Marble detection through RFID systems, exploiting the interaction between stones and RF radiations, has been preliminary discussed by us in [13] and will be further detailed in the following Section 3 to Section 8.

Also the techniques proposed in $[5-8,14,15]$ aim at classification and fine-grain analysis of the texture and surface of stone slabs in a static and controlled environment, with conditions different from those found inside a marble machine. Moreover, for marble machines a simpler on/off detection is required, but with the possibility to be performed in real time, with higher robustness, to be easily integrated with the machine controlling system, having low maintenance costs. Finally, the computational power needed to implement computing techniques based on wavelets, Gabor filters, or neural networks as in $[6,7,14]$ is not compatible with the utilization of the PLC devices commonly used in marble machines controlling systems, above all if such techniques must be applied in several points inside the machine.

To address some of the issues of the state-of-the-art slab detection, in this paper we present the experimental characterization of 4 different RFID systems and of 2 capacitive sensing systems in the detection of the presence of the marble slab outside and/or inside the marble machine. Our work aims at multipoint sensing scheme being contactless, nearly maintenance-free, operating in real-time, robust to harsh environment conditions. The detection aims at providing the machine controlling system with an on/off information concerning the presence of the marble slab under the working tools and is not intended to provide low-scale information on the properties of the material-like composition, thickness, unhomogeneousness due to small cracks, and so forth.

The target of the work, concerning the RFID and capacitive wireless sensing technologies, is highlighting the advantages and the limits of them when applied to detection tasks, mainly in the marble industry process control, and suggesting which technologies are most suitable and how they can be used.

\section{RFID for Process Control in Marble Machines}

RFID is a mean of identifying, but also tracking and detecting, an item using radio frequency communication, which takes place between a transmitter, usually called "reader," and a transponder (silicon chip connected to an antenna), usually called "tag." The physical coupling is based on magnetic or electromagnetic fields. Tags can either be passive, that is, powered by the reader field, semipassive or active, that is, 


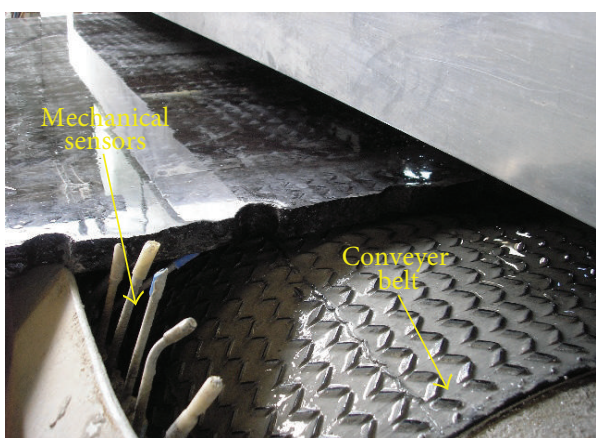

(a)

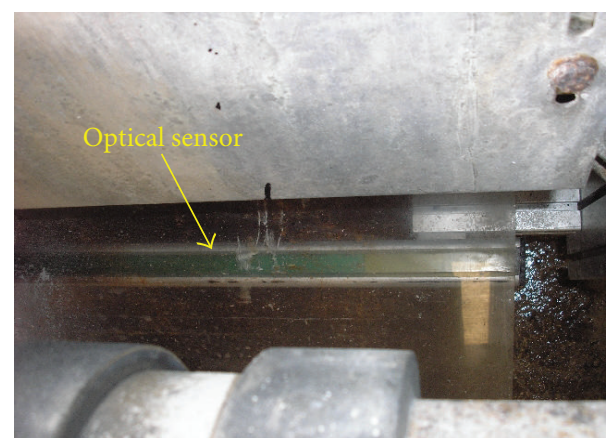

(b)

Figure 3: Mechanical sensors and optical sensors at the machine entrance stage.

powered by a battery; in this last case the transmitter is usually the tag itself [16].

At the state-of-the-art the application of RFID technologies has been analyzed and implemented for logistics (for which commercial solutions are available, as an example in the marble industry $[17,18]$ using $13.56 \mathrm{MHz}$ passive tags and handheld RFID reader), for the management of production [19, 20], and even for localization [21], but less for industrial machine process control and sensing.

In marble machines, the effects on reader-tag communication depend on various parameters: the composition and shape of the stone slab, the operating frequency and power levels of the RFID system, the radiation pattern of the antennas, the distance between tag and reader and their relative orientation, and the working environment (presence of water or dust or a mixture of both, presence of metallic planes in the machine, and composition of the conveyer belt). Hence an experimental test campaign on real case studies using different RFID systems is required. Such experimental campaign, missing in the literature, is the main objective of the present part of the work on RFID technologies.

Since marble machines are not produced in large numbers, therefore not justifying the development of ASICs, in our experiments we implemented 4 RFID systems starting from commercially available tags and readers and customizing the hardware components and/or the relevant software for proper configuration of the experimental setup and for acquisition and processing of test results. Due to the poor availability of experimental data in the literature, we have investigated the application of RFID systems ranging from the low frequencies (LF) to the ultrahigh frequencies (UHF) bands. The four considered RFID systems are shown in Figure 4. The low-frequency RFID system of Figure 4(a) [22] uses passive tags and a communication frequency of $125 \mathrm{kHz}$; the high-frequency (HF) system of Figure 4(c) at $13.56 \mathrm{MHz}$ uses passive tags [23]; two ultrahigh-frequency systems, at $868 \mathrm{MHz}$ [24], see Figure 4(b), and at $2.45 \mathrm{GHz}$ [25], see Figure 4(d), use, respectively, passive tags and active tags.

The following experiments and related hardware and/or software customizations of the systems in Figure 4 have been realized: (i) measurement and comparison with a given threshold of the amplitude of the signal modulated by the tag and decoded by the reader; (ii) proper setting, through the control software, of the power radiated by the reader antenna; (iii) detection and comparison with a given threshold of the packet reception error rate in the tag-reader communication.

The above solutions are alternatives of each other, and the most suited depends on the possibility of configuration offered by RFID components. As an example in the considered systems $[24,25]$ the reader power level may be configured. The software of [25] allows processing of communication data to determine the packet error rate. For the test campaign sixteen stone samples, different in size and shape (typically rectangular with the larger sizes in the order of some tens of $\mathrm{cm}$ and height of up to a few $\mathrm{cm}$ ) and representative of the possible materials processed in marble industry (e.g., onyx, marble, granite, etc.), have been considered. In Figure 5 six of the sixteen stone samples are shown.

\section{Experimental Analysis of the LF RFID System}

The $125 \mathrm{kHz}$ LF RFID system has been firstly characterized in a test setup reproducing the environment inside the marble machine; as sketched in Figure 6 the passive tags have been embedded in fixed positions in the conveyer belt which is made of plastic/rubber and is transparent to LF (the scheme in Figure 6 realizes a smart conveyer belt) or placed under it. The aim of the first test has been the determination of the maximum distances in the $3 \mathrm{D}$ space where the tags are detected by the reader without the interposition of the stone samples. In this test the observed output is the data output of the RFID LF reader, see Figure 7 , sent to a PC connected to the reader and shown by means of an application providing on/off detection information (plus the code of the tag in case of detection). The LF reader, using a coil antenna with a diameter of about $20 \mathrm{~cm}$ (see Figure $4(\mathrm{a})$ ), communicates with passive tags of size $8 \mathrm{~cm} \times 5 \mathrm{~cm}$ through inductive coupling. The output power of the reader is $100 \mathrm{~mW}$. Figure 7 also shows the schematic waveform of the signal on the reader coil, highlighting the carrier and its modulation (communication data).

The experimental results, reported in Figure 8, show that the tags can be detected up to a distance of roughly $20 \mathrm{~cm}$ in the $3 \mathrm{D}$ space. The experiment has been repeated with all 


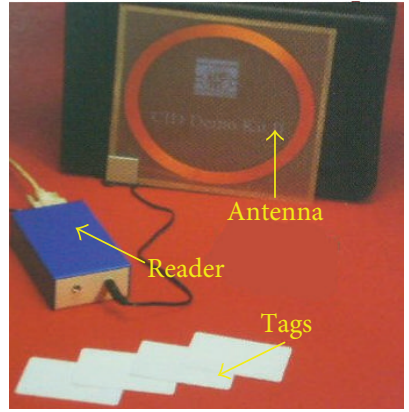

(a) $\mathrm{LF} 125 \mathrm{kHz}$

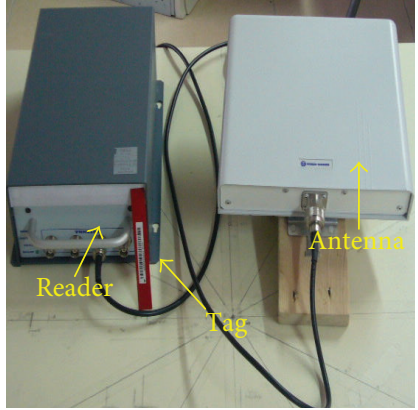

(b) UHF $868 \mathrm{MHz}$

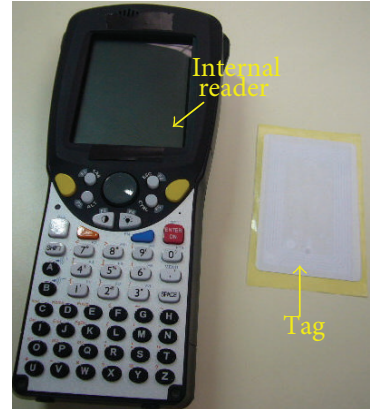

(c) $\mathrm{HF} 13.56 \mathrm{MHz}$

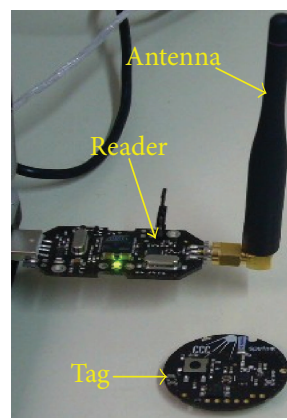

(d) UHF $2.45 \mathrm{GHz}$

Figure 4: Considered RFID systems (reader, antenna, and tag).
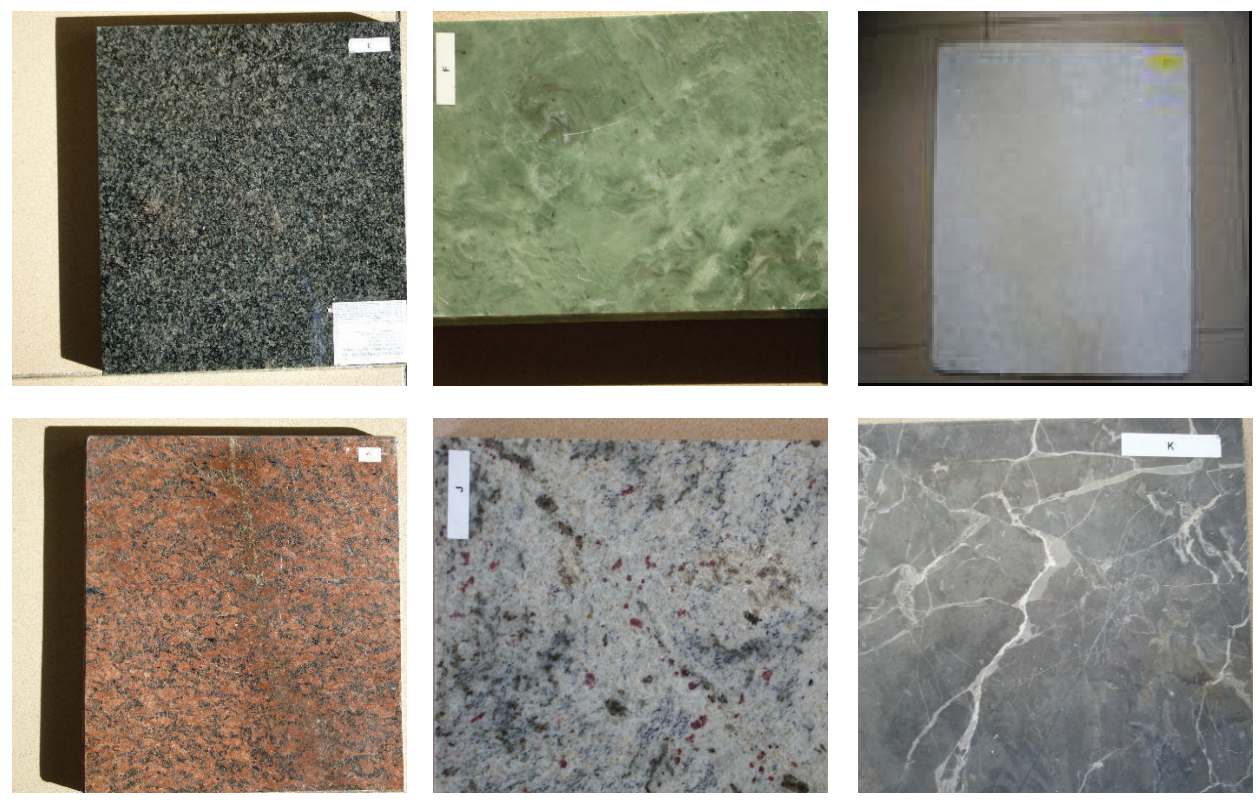

Figure 5: Six of the 16 marble slabs used for the test campaign.

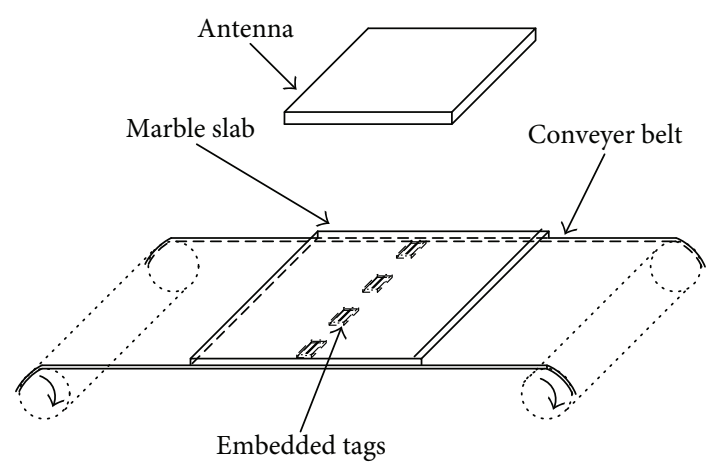

FIGURE 6: Test configuration with tags embedded in the conveyer belt.

the different stone samples interposed between the tags and the reader coil antenna, with and without the presence of a water layer of some $\mathrm{cm}$ and with and without the presence of marble dust and mud. The obtained results are substantially the same of those reported in Figure 8, in which only results on the $Z Y$ plane are reported, due to the circular symmetry of the antenna lying on the $X Y$ plane.

Experimental results prove that, as expected at the test frequency, stones, water, and mud are almost transparent to LF radiations. Finally we repeated all the above tests analyzing the analog decoded signal in the LF reader, see Figure 7, considering a fixed distance of $10 \mathrm{~cm}$ (compliant with the use of the system inside the machine) between the nearest tag and the reader coil antenna. The analyzed signal is obtained through a custom circuit that we added to the reader circuitry in order to filter and measure the amplitude of the decoded signal (envelope of the received signal). Results of these tests are showed in Figure 9. Each reported measurement is generated by averaging ten measurements of the peak-to-peak signal amplitude, with and without interposition of the stone slab between the tag and the reader. The peak-to-peak voltage level measured in absence of stone samples resulted to be $120 \mathrm{mV}$. Repeating the tests with the stones interposed (more 


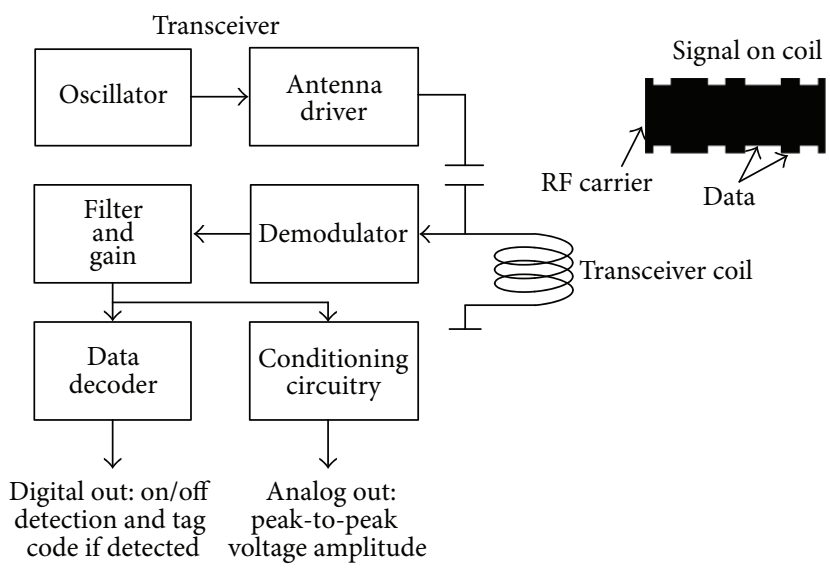

FIGURE 7: Architecture of the reader, LF RFID system, and schematic illustration of data modulation.

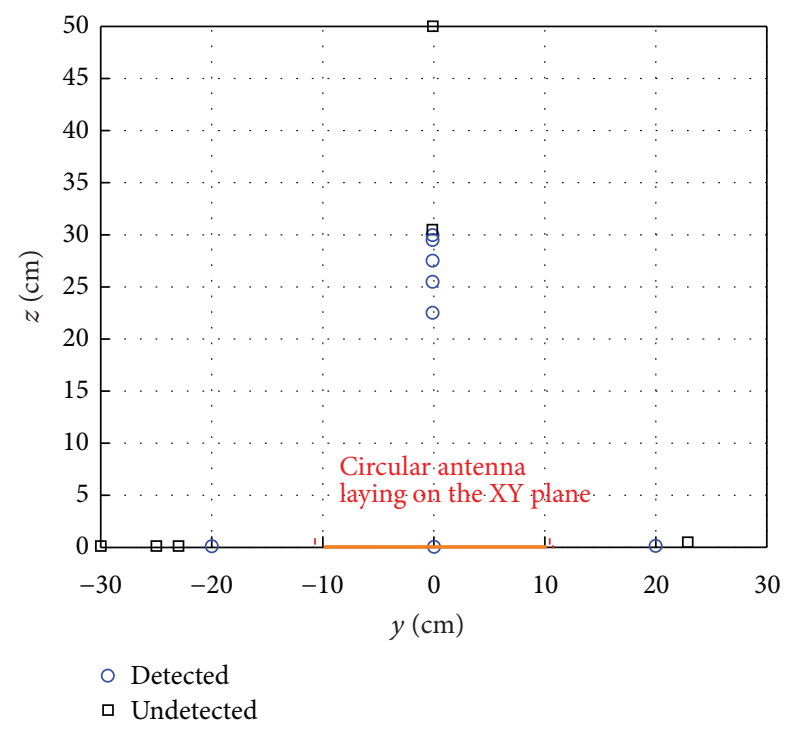

FIgURE 8: Experimental points of detection, LF RFID system.

than 16 different stone samples labeled with letters from A to $\mathrm{P}$ in Figure 9, with/without water or mud or marble dust), the revealed signal ranged between $115 \mathrm{mV}$ and $125 \mathrm{mV}$ with small differences (within $\pm 4 \%$ ) versus the $120 \mathrm{mV}$ reference (see Figure 9). Since the measured value slightly depends on the specific type of stone the above detecting technique could possibly be used for industrial applications involving a specific stone type but not in a machinery where the type of stone samples varies from slab to slab.

To solve this issue for the LF RFID system a different detecting strategy has been adopted: instead of embedding the tags in the conveyer belt as in Figure 6, the tag has been applied on the side surface of each marble slab through a fast dry resin as in [17]. The tag is not placed on the top surface of the marble slab in order to avoid any damage by the polishing abrasive heads. The tag has in this case the function of confirming the presence of the slab on the conveyer belt in the position expected by the PLC. This configuration allows the detection, even in presence of water and dirt, of the tag

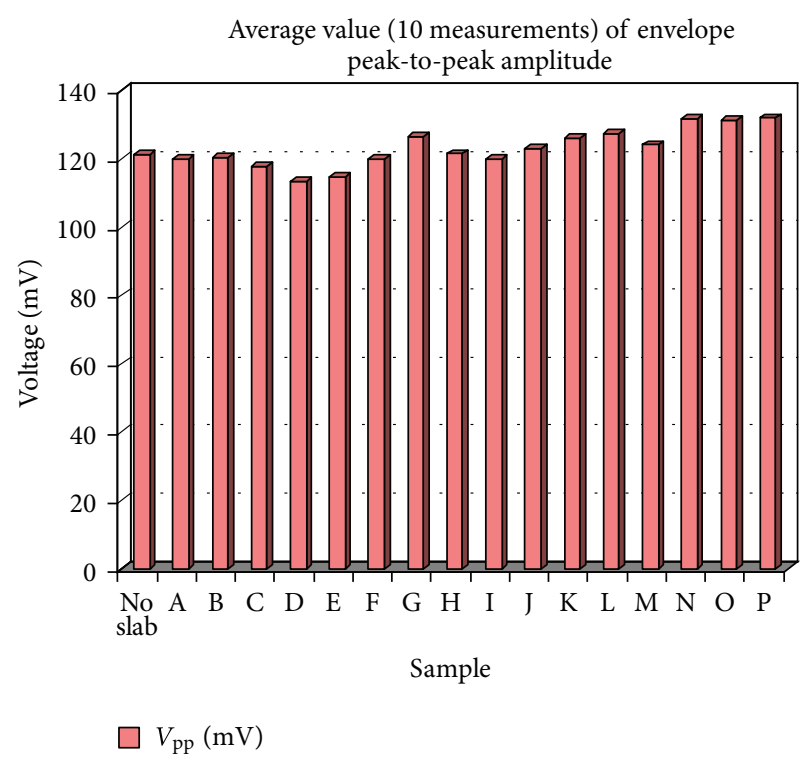

FIGURE 9: Peak-to-peak amplitude of the decoded signal picked up on the reader by a custom circuitry.

and of the corresponding marble slab when the tag is passing in the area covered by the reader antenna. For the considered LF system the antenna-tag distance should be within $15 \mathrm{~cm}$. Such distance allows the application both outside and inside the machine.

\section{Experimental Analysis of the HF RFID System}

For the RFID HF system in Figure 4(c), communicating through magnetic coupling with passive tags at $13.56 \mathrm{MHz}$, by means of a Windows CE application on the handheld device we implemented similar tests and obtained similar results as in the case of the RFID LF system. Figure 10 shows the experimental results concerning the detection limit points in the 3D space without any stone sample interposition. The only difference with the LF system is that the HF system is based on a handheld battery-powered device [23] with an internal tag reader and an internal antenna. Due to the limited radiated power, in the range of tens of $\mathrm{mW}$ (typical of HF RFID readers for handheld applications $[26,27])$, the maximum reachable distance is below $8 \mathrm{~cm}$. Using an HF RFID reader, not battery powered, a higher power level could be irradiated and hence we expect the achievement of performances similar to those of the LF system concerning the maximum distance of tag reading. As an example, the RFID HF reader [28] technical specifications report a maximum output power of about $4 \mathrm{~W}$ and a tag reading distance above $80 \mathrm{~cm}$.

\section{Experimental Analysis of the UHF RFID System}

For the RFID UHF system, shown in Figure 4(b) and working at $868 \mathrm{MHz}$, we have firstly repeated the characterization with the test setup in Figure 6 reproducing the marble machine 


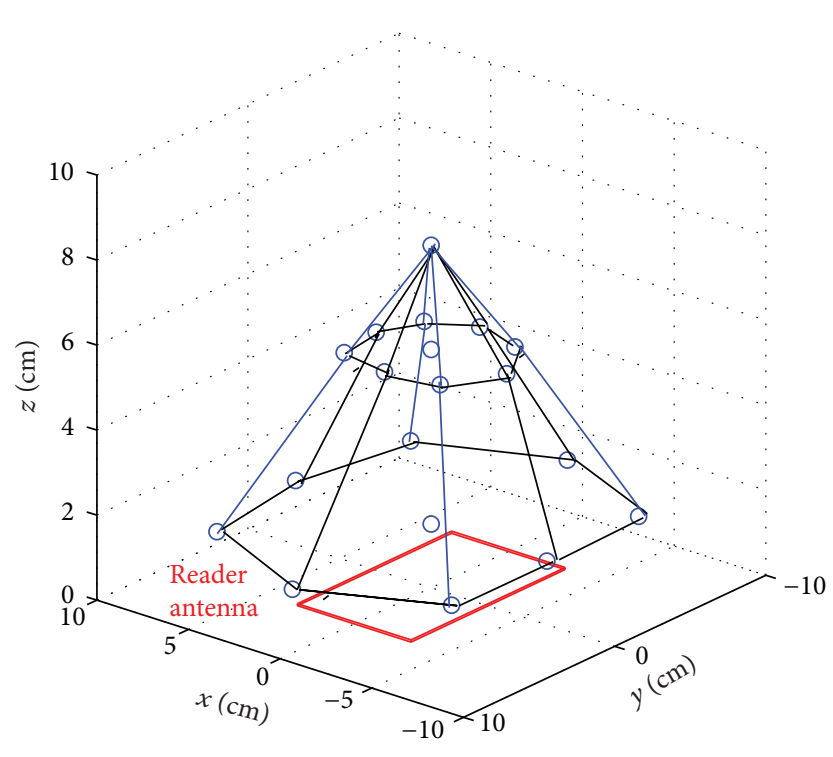

Detection

FIGURE 10: Experimental surface of maximum detection distance, HF RFID system.

working environment, embedding the passive tags in fixed positions in or below the conveyer belt. The aim of the first test was the determination of the maximum distances in the $3 \mathrm{D}$ space at which the tags are detected by the UHF reader for a defined tag-antenna relative orientation, without interposition of stone samples, at different radiated power levels. Experimental results are reported in Figure 11 for the case example of the reader radiating a $100 \mathrm{~mW}$ power. At UHF frequencies the coupling between RFID reader and tags is electromagnetic.

The power level irradiated by the UHF reader antenna is programmable in the range [ $100 \mathrm{~mW}, 4 \mathrm{~W}]$. To be noted is that power regulations in Europe are characterized by a limit of $500 \mathrm{~mW}$ while the maximum level of $4 \mathrm{~W}$ is permitted by US regulations.

Besides the irradiated power level also the working frequency of the selected UHF reader [24] is programmable in order to support both European and US regulations. The used tags are passive devices compliant with the ISO180006B standard and compliant with both US and European regulations concerning frequency and power levels. The antenna is a planar one with a wide frequency working range from roughly $800 \mathrm{MHz}$ to $960 \mathrm{MHz}$. To carry out the test campaign, the Microsoft Visual C++ PC application controlling the UHF reader has been modified and customized in order to provide an interface suitable for testing purposes. In Figure 12 the customized graphical user interface (GUI) is shown. Figure 13 reports the maximum distance at which the tag is still detected as a function of the reader antenna power level.

The experiment has been repeated with all the different stone samples interposed between the tags and the reader antenna, with and without the presence of a water level of few $\mathrm{cm}$ and with and without the presence of marble dust

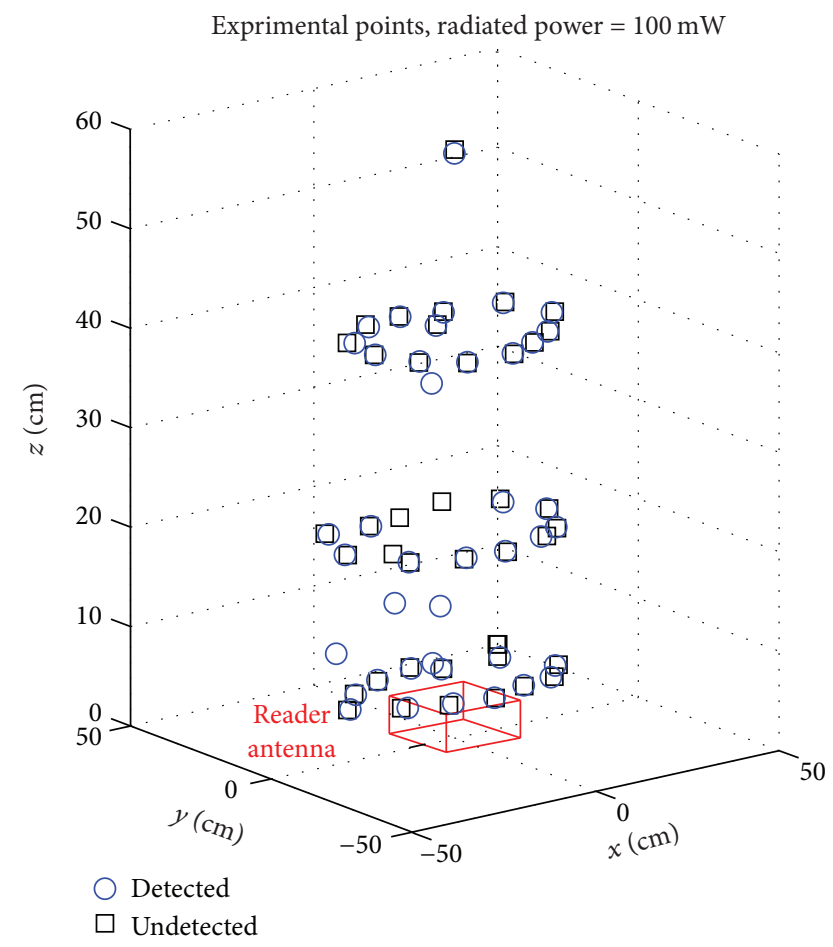

FIGURE 11: Experimental points of detection, UHF RFID system, for a radiated power of $100 \mathrm{~mW}$.

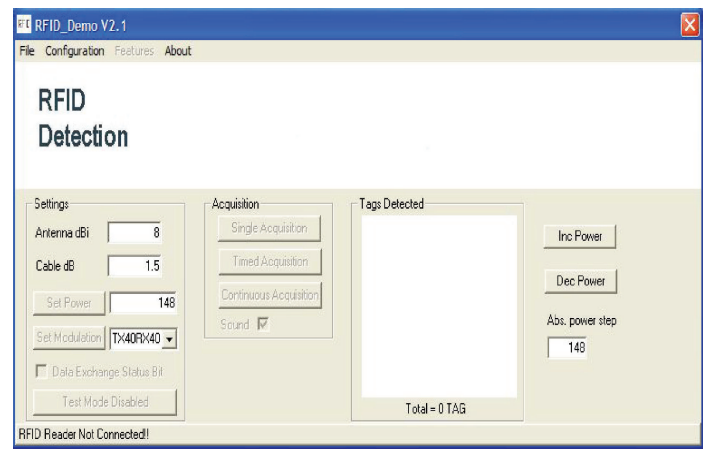

FIGURE 12: The customized GUI of the application for the UHF reader control.

and mud. Differently from what measured in the case of LF and HF systems, at UHF frequencies the communication is completely shielded by water, that is, in presence of a thin layer of water, or in some cases just with a few water drops wetting the tag, the tags are not detected. These experimental results are aligned with studies [29] on water properties, see Figure 14, proving that in the UHF range the intensity of a plane incident wave decreases to $1 / e$ (i.e., $63 \%$ absorbed) in a penetration distance of about $1 \mathrm{~cm}$ or lower depending on the test conditions (note that in our application the water layer can be of some $\mathrm{cm}$ ). Therefore UHF systems cannot be used inside the marble machine.

The results of the test campaign applied using the configuration of Figure 6 with different types of stones interposed (in a dry environment) prove that at UHF frequencies a stone 


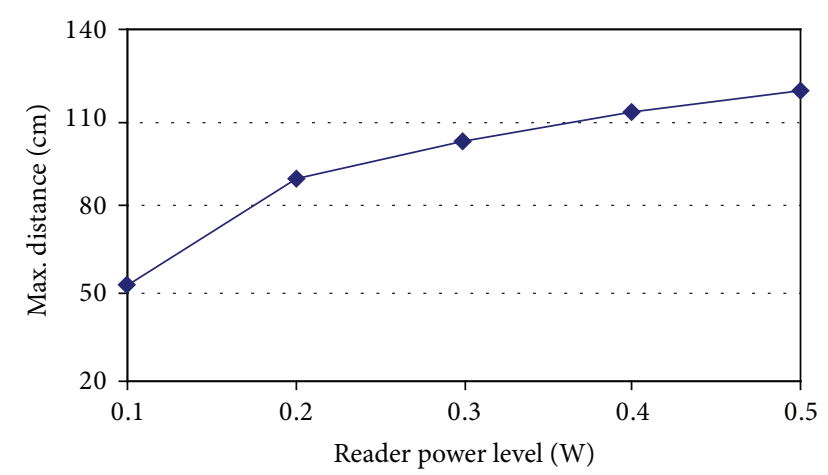

Figure 13: Max. distance of tag detection versus radiated power.

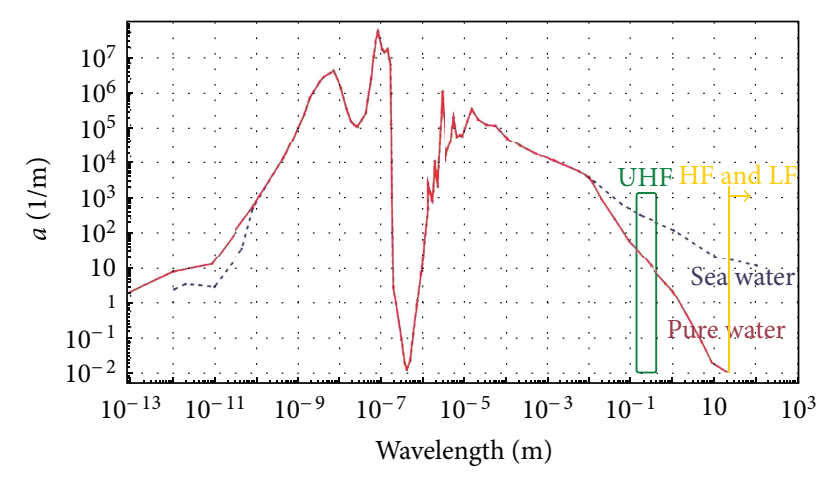

FIGURE 14: Water absorption spectrum.

sample attenuates the RF signal. Therefore the tags embedded in the conveyer belt can be detected or not depending on the distance and power level radiated by the reader.

According to this result the detection of the marble slab with UHF systems is possible following the strategy described hereafter. First, as sketched in Figure 6, the tags should be embedded in the conveyer belt while the reader antenna should be attached on the upper part of the machinery in a fixed position (e.g., $40 \mathrm{~cm}$ above the conveyer belt in our case study). Secondly, the emitted power level should be properly configured so that (a) when the stone sample is interposed between the tag and the reader antenna the RF signal is attenuated under a certain bound and the tag cannot be detected by the reader; (b) when the stone sample is not present the tag communicates correctly with the reader. After the test campaign in a dry environment we determined that configuring the UHF reader with a radiated power ranging from about $400 \mathrm{~mW}$ to $600 \mathrm{~mW}$ the detection of stone samples is allowed according to the above on/off strategy. The experimental results of this test are reported in Figure 15 for a case study of slabs sized about $30 \mathrm{~cm} \times 30 \mathrm{~cm}$. Hence configuring the reader with a proper power level in this range (e.g., $500 \mathrm{~mW}$ ) and embedding the tags in the belt, a RFID UHF system can be used for automatic detection in the process control of marble industry. It must be pointed out that this type of detection is possible only in a dry environment; therefore it cannot be used inside the machine where a water layer of several millimeters is always present.

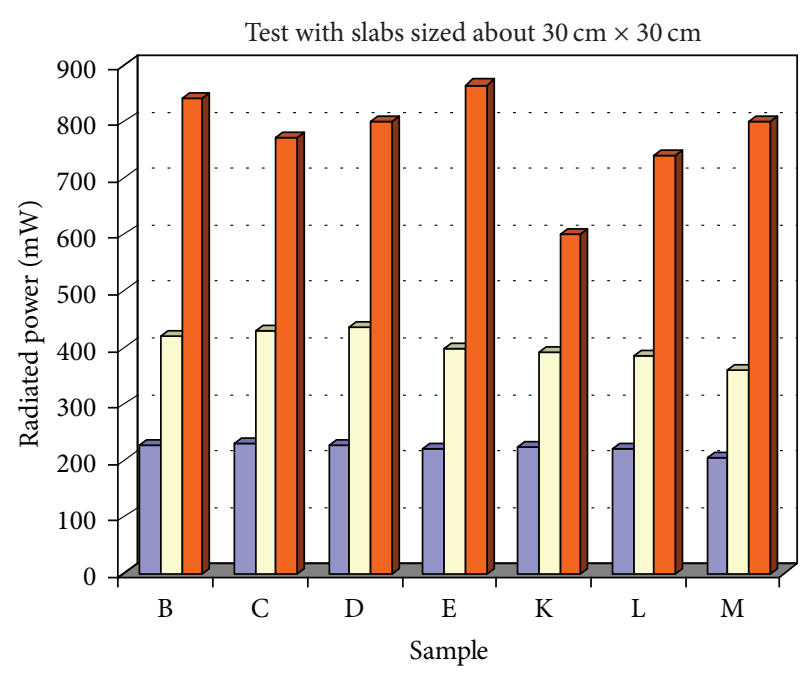

$\square$ Minimum detection power (mW), no slab, no conveyer belt $\square$ Maximum non-detection power ( $\mathrm{mW}$ ), no slab, with conveyer belt $\square$ Minimum detection power (mW), with slab and conveyer belt

FIGURE 15: Experimental results showing the radiated power in the on/off detection strategy test.

On the contrary, outside the machine, in a dry environment, the UHF RFID system represents an interesting alternative to traditional mechanical and optical systems to detect the presence of the marble slab at the entrance of the machine. With respect to mechanical sensors, the UHF RFID systems benefit of being contactless. With respect to optical systems the UHF RFID solution is more robust to the presence of a dirty environment.

\section{Experimental Analysis of the Microwave Active RFID System}

For the RFID UHF system at $2.45 \mathrm{GHz}$ we implemented tests similar to those carried out for the RFID system at $868 \mathrm{MHz}$. One of the main differences is represented by the fact that the $2.45 \mathrm{GHz}$ solution, shown in Figure 4(d), uses active tags and its maximum detection distance, without the marble slab, is up to $20 \mathrm{~m}$ at $1 \mathrm{~mW}$ of effective radiated power. Since the distance to be covered in marble machine applications is below $50 \mathrm{~cm}$, during the tests we configured the $2.45 \mathrm{GHz}$ RFID tags for $1 / 16 \mathrm{~mW}$ effective radiated power in order to limit the operating range at a few tens of $\mathrm{cm}$ when the marble slab is not present. For the $2.45 \mathrm{GHz}$ active system, the slab detection strategy is not based on the reception/no reception of data packets transmitted by the active tag but on the measure of the reception error ratio and its change in the two cases of slab interposed and not interposed between the tag and the receiver antenna. Reception errors are due to RF absorption and/or scattering by the stone. In the following, the reception ratio refers to the number of correctly received packets divided by the number of expected (i.e., transmitted) packets. For the considered $2.45 \mathrm{GHz}$ RFID system, a data packet is transmitted every about $250 \mathrm{~ms}$ (4 packets per second); transmission power is cyclically changed every data 


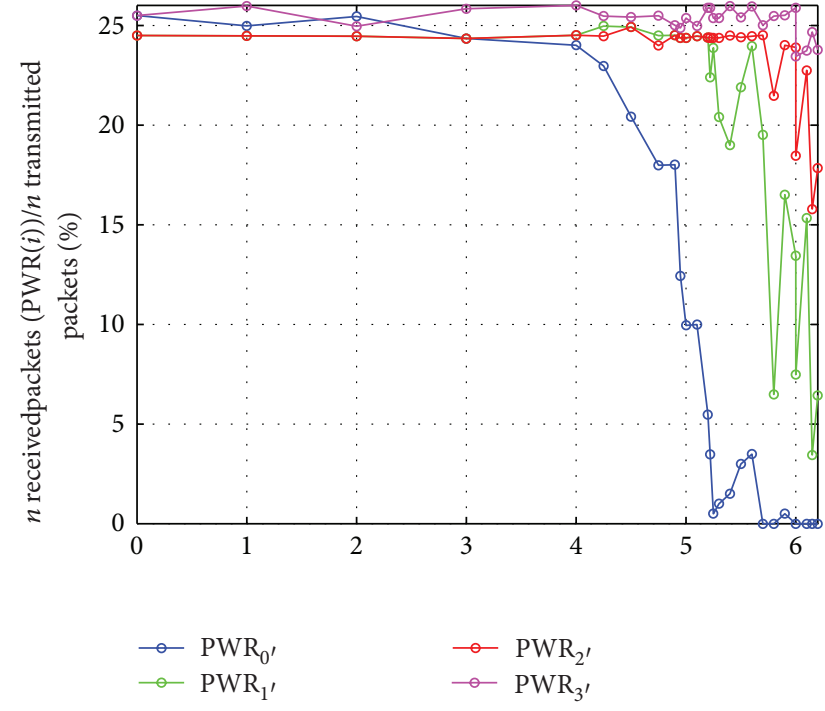

FIGURE 16: Reception ratio versus tag-reader distance for the $i$ th emitted power level $(1 / 64 \mathrm{~mW}, 1 / 16 \mathrm{~mW}, 1 / 4 \mathrm{~mW}$, and $1 \mathrm{~mW}$, resp., $\mathrm{PWR}_{0}, \mathrm{PWR}_{1}, \mathrm{PWR}_{2}$, and $\mathrm{PWR}_{3}$ ).

packet in the set of 4 different radiated power levels: $1 / 64 \mathrm{~mW}$, $1 / 16 \mathrm{~mW}, 1 / 4 \mathrm{~mW}$, and $1 \mathrm{~mW}$. Obviously the reception error rate can be calculated as 1 minus the reception rate of correct data packets.

In order to collect experimental data, we developed an ad hoc Matlab (from the Mathworks) application on a PC processing data received by a USB $2.45 \mathrm{GHz}$ receiver. Packets received with errors are discarded and therefore counted as nonreceived. The original firmware $(\mathrm{C})$ on the tag and on the receiver was customized in order to format data packets according to data processing needs.

Preliminary tests were carried out in order to confirm that with transmitter and receiver placed at a short distance the reception rate was $100 \%$ for each power level, therefore excluding buffer overflow problems on the receiving PC (e.g., due to application overhead problems). To be noticed is that the packet distribution is not exactly $25.0 \%$ for each power level, but about $25.5 \%, 24.25 \%, 24.5 \%$, and $25.75 \%$ for packets at $1 / 64 \mathrm{~mW}, 1 / 16 \mathrm{~mW}, 1 / 4 \mathrm{~mW}$, and $1 \mathrm{~mW}$ power, respectively, as measured in several experiments and in conditions of no packet loss. Figure 16 shows the reception ratio in percentage for each power level as a function of the tag-reader distance, measured in a first experiment carried out in a mixed indoor/outdoor environment.

For the power of $1 / 64 \mathrm{~mW}$, Figure 16 shows a monotonic decrease and a knee in the curve of reception ratio versus distance. Curves at different power levels have not exactly the same trend of the curve for the power of $1 / 64 \mathrm{~mW}$, probably due to reflections and/or other scattering effects in the working environment, that should of course be characterized and/or avoided in on-field applications.

To achieve the best sensitivity in the reception ratio, the experimental setup for slab detection was arranged by properly displacing tag and receiver antenna so that the reception ratio without the slab was below $100 \%$, ideally on
TABLE 1: Relative dielectric permittivity of different materials.

\begin{tabular}{lc}
\hline Material & Dielectric permittivity, $\varepsilon_{r}$ \\
\hline Bianco Carrara & 5.7 \\
Rosa Portogallo & 6.8 \\
Pietra di Trani & 13.8 \\
Granito Grigio & 29.9 \\
Pietra del Cardoso & 9.2 \\
Ceramics & $4.3-6.7$ \\
Plastic (PVC, Plexiglass) & $2-3$ \\
\hline
\end{tabular}

a working point in the decreasing part of the curve after the knee, in order to have a good sensitivity of the reception ratio versus slab interposition.

During tests with the sixteen slabs we carried out about five measurements of the error ratio just a few seconds before any slab interposition in order to avoid drifts due to possible environmental effects and then during slab interposition. The two sets of measured values are reported in Figure 17, linked by a line identifying the progression of the experiment. With this configuration, when the marble slab is not passing, the reception ratio is from $40 \%$ to $80 \%$ while with slab interposition, in most cases, the reception ratio drops below $10 \%-20 \%$ (i.e., the reception error rate is up to $80 \%-90 \%$ ); this decrease is interpreted as detection of the stone sample, but in some cases (slab B, E, J, L) the presence of the slab causes an increase of the detection ratio instead of a decrease. Since the behavior of the system depends on the specific type of stone, the described detecting technique could possibly be used only for applications involving a specific stone type but not in a machine where the type of stone sample is not known a priori.

To be noted is that the considered $2.45 \mathrm{GHz}$ RFID system has two main disadvantages:

(i) its use is possible only at the entrance of the marble machine in a dry environment since, as in case of the $868 \mathrm{MHz}$ RFID system of Section 6, also microwave signals are absorbed by water;

(ii) the use of active tags causes a higher cost for the tags. Therefore the solution with passive tags should be preferred for process control applications in the marble industry.

\section{Capacitive Sensing Principle and Front-End Circuitry for Marble Detection}

To address some issues of the currently used and of other proposed slab detection techniques, this section of the work proposes novel capacitive sensors for the detection of stone samples during the industrial process, inside and outside the machine, and by means of contactless and low-complexity devices. Indeed, as reported in Table 1, the stone samples used in the marble industry have relative dielectric permittivity $\varepsilon_{r}$ in the range 5 to $30[5,9,10]$ that can be used to reveal the presence of a stone sample being different from those of air $\left(\varepsilon_{r}=1\right)$, water $\left(\varepsilon_{r}=81\right)$, or plastic ( $\varepsilon_{r}$ between 2 and 3$)$. 


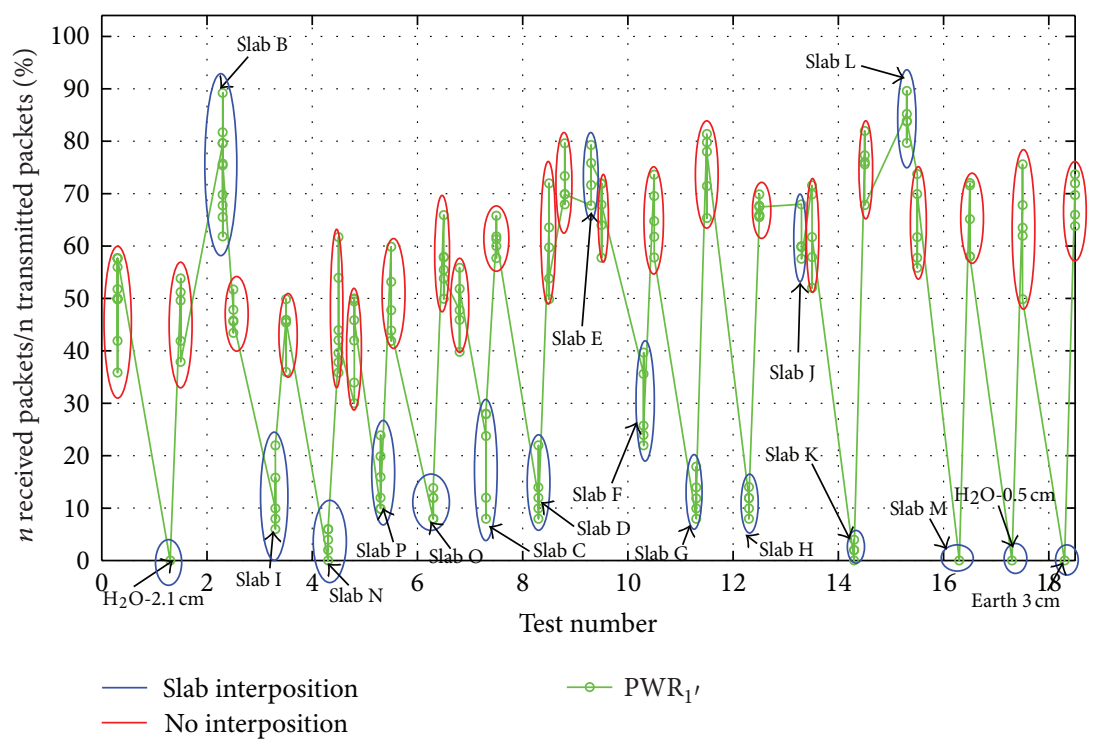

FIGURE 17: Reception ratio normalized to $100 \%$ measured with/without slab interposition for radiated power of $1 / 16 \mathrm{~mW}$.

As mentioned in Section 2, capacitive sensors have been studied in the literature [9] but the solutions proposed are optimized for a fine-grain analysis of the porosity and surface defects of the stone material in a controlled working environment (dry and clean) rather than the real-time detection of a marble slab in an industrial machine. Furthermore in [9] the realized sensor should be taken in contact with the stone sample with a controlled force of $1 \mathrm{~N}$ and the stone sample should be held in a fixed position. Instead the aim of our work is the contactless detection of the presence of stone samples moving on the conveyer belt and considering a real industrial environment characterized by the presence of mud, water, and stone residuals and is not intended to measure or give information about material properties (e.g., thickness, composition, unhomogeneousness, etc.).

The basic scheme exploited in this work is the capacitor with parallel metallic plates filled with multiple layers of different dielectric materials; see Figure 18 for the schematic representation in case of a number of dielectric layers $n=3$.

The value of the capacitance can be determined considering the series of $n$ capacitors each of value $C_{i}=\varepsilon_{o} \cdot \varepsilon_{r i} \cdot S / d_{i}$ with $i=1, \ldots n$, being $S$ the area of the metallic plates, and $d_{i}$ and $\varepsilon_{r i}$ the thickness and the relative dielectric permittivity of each layer.

Firstly we realized a capacitive sensor with plates sized $6.5 \mathrm{~cm} \times 12.5 \mathrm{~cm}$ ( $S$ roughly $\left.80 \mathrm{~cm}^{2}\right)$ in a test-bed reproducing a marble machine working environment found at the machine entrance stage, without the presence of water (note that the characterization of the sensing system with a test-bed considering the presence of water will be discussed in Sections 9.1 and 9.2). The total distance $D$ between the plates has been fixed at $4.5 \mathrm{~cm}$ to allow the contactless interposition of different stone samples which have a typical thickness between 1 and $3 \mathrm{~cm}$. The reported analysis is still valid for case studies with maximum thickness higher than $3 \mathrm{~cm}$ by increasing, accordingly, the distance $D$ between the

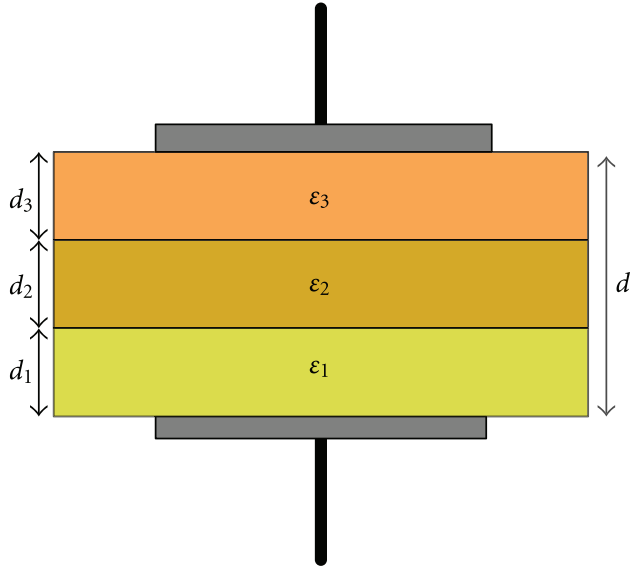

FIGURE 18: Multidielectric layer capacitive sensor.

plates. Mounted at the entrance of the marble machine, the capacitance value, when the marble sample is not present, is expressed by (1) and amounts to about $1.6 \mathrm{pF}$ :

$$
C_{0}=\varepsilon_{o} \cdot \frac{S}{D}
$$

In this case the filling dielectric is just air. When a stone sample of relative dielectric permittivity $\varepsilon_{r}$ and thickness $x$ is passing through the metallic plates, see Figure 19, the capacitor value $C_{\text {sens }}$ is expressed by (2), and the ratio $C_{\text {sens }} / C_{0}$ is expressed by (3):

$$
\begin{aligned}
C_{\text {sens }} & =\varepsilon_{o} \cdot \varepsilon_{r} \cdot \frac{S}{\left[x+\varepsilon_{r}(D-x)\right]}, \\
\frac{C_{\text {sens }}}{C_{0}} & =\varepsilon_{r} \cdot \frac{D}{\left[x+\varepsilon_{r} \cdot(D-x)\right]} \\
& =\frac{\varepsilon_{r}}{\left[x / D+\varepsilon_{r} \cdot(1-x / D)\right]} .
\end{aligned}
$$




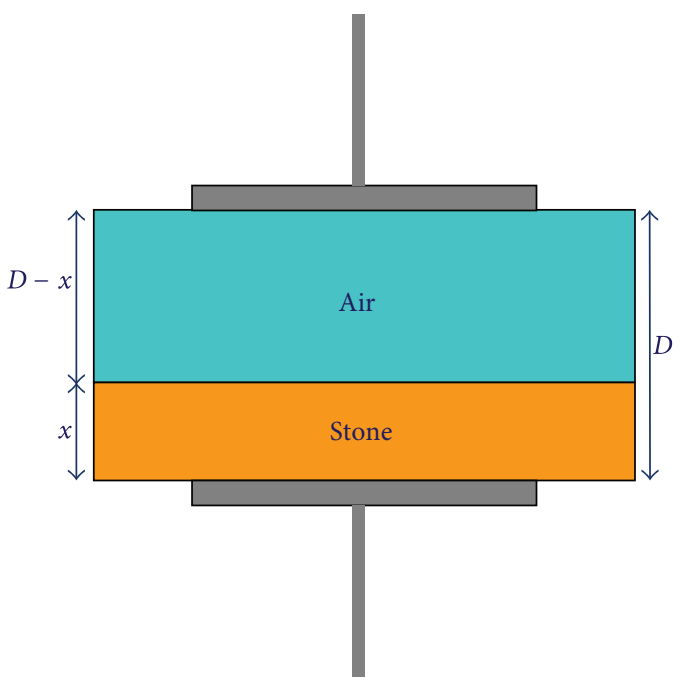

FIGURE 19: Multidielectric layer (air/stone) capacitive sensor.

For typical stone samples $\varepsilon_{r}$ ranges from 5 to 30 , see Table 1 , and $x / D$ ranges from 0.25 to 0.75 for the selected value $D=4.5 \mathrm{~cm}$. Therefore, when a stone sample is passing, the capacitor value is increased with a ratio $C_{\text {sens }} / C_{0}$ expressed by (3). As a consequence, the detection of the stone sample can be simply realized by revealing a change in the capacitor value higher than a given threshold. The easiest way $[30,31]$ to reveal this change is inserting the capacitive sensor in an astable circuit and measuring the shift of the oscillating frequency with a microcontroller-based circuit, as shown in Figure 20. The astable circuit is realized with a simple 555 IC and has a theoretical oscillating frequency expressed by

$$
F=\frac{1}{\left[\ln (2) \cdot\left(R_{A}+2 R_{B}\right) \cdot\left(C_{\text {sens }}+C_{p}+C_{\text {ext }}\right)\right]} .
$$

In (4) $C_{\text {sens }}$ refers to the capacitive sensor, $C_{p}$ to the open-circuit parasitic capacitor due to wire connections and astable component input capacity, and $C_{\text {ext }}$ to an external capacitor inserted to set at a desired value $F_{0}$ the oscillation frequency when $C_{\text {sens }}=C_{0}$. This way the capacitance change is transformed in a frequency change, easily revealed through a low-cost low-power [32] 8-bit microcontroller.

With marble interposition the value of $C_{\text {sens }}$ in (2) increases and hence the oscillation frequency in (4) decreases. To be noticed is that the size of the surface plates $S$ does not influence the change of the capacitance ratio $C_{\text {sens }}=C_{0}$; the value of $S$ is important to determine absolute values of $C_{\text {sens }}$ and $C_{0}$ giving measurable variation in (4).

After realizing the circuit we implemented a test campaign using 16 stone samples, different in size and shapes (typically rectangular with horizontal size in the order of several tens of $\mathrm{cm}$ and height of up to a few $\mathrm{cm}$ ), representative of the possible materials processed in marble industry (e.g., onyx, marble, and granite). Snapshots of 6 of the 16 different stone samples used during the testing campaign are reported in Figure 5.

The obtained results are summarized in Figure 21 which reports the frequency change with respect to $F_{0}$ for each stone sample (labeled with a letter in the range $[\mathrm{A}, \ldots, \mathrm{P}])$. The frequency changes in Figure 21 range from a minimum of $2.6 \mathrm{kHz}$ (sample $\mathrm{K}$ in Figure 21) to a maximum of $26 \mathrm{kHz}$ (sample I in Figure 21) allowing a reliable detection of the stone presence. The astable circuit reference frequency is set at $F_{0}=219 \mathrm{kHz}$, see in Figure 22 the snapshot of the signal generated by the circuit in Figure 20 when $C_{\text {sens }}=C_{0}$. The tests have been carried out also in the presence of mud, marble dust, and powder, obtaining results similar to those in Figure 21.

In the test campaign, we used a threshold for frequency change detection of $1 \mathrm{kHz}$ which ensures a margin of $1 \mathrm{kHz}$ against false detections and higher than $1.6 \mathrm{kHz}$ (the minimum $2.6 \mathrm{kHz}$ decrease when a stone is present minus $1 \mathrm{kHz}$ threshold) against missed detections. Since our goal is an on/off detection, the above margins are sufficient to avoid errors caused by changes in the reference frequency during the time occurring for a typical slab to pass under the sensor (up to tens of seconds); such reference frequency changes can be caused by parasitic capacitance change, deposition of marble dust or mud, and change of temperature conditions. To face slow variations of the reference frequency, the microcontroller via software can monitor $F_{0}$ and manage adaptive thresholds.

It is worth noting that in our proposed front-end circuitry the capacitance change (due to the stone slab presence) is detected through a frequency change with respect to the fixed frequency value $F_{0}$. To further increase the robustness of our system to electromagnetic interference (EMI), typical of industrial environments, a variable resistor $R_{B}$ can be used in the scheme of Figure 20 to tune the value of $F_{0}$ according to (4). This way when applying the proposed sensing system in a specific industrial environment during the system calibration phase the frequency $F_{0}$ can be set in a range where the EMI is null or minimal by changing the value of $R_{B}$. To this aim Figure 23 shows that to change $F_{0}$ in the range $[100 \mathrm{kHz}-500 \mathrm{kHz}]$ it is enough to change $R_{B}$ in the range $[5 \mathrm{k} \Omega-100 \mathrm{k} \Omega$ ]. These values have been sized under the hypothesis, which is always verified in our experimental tests and in industrial systems we considered, that in the selected range $[100 \mathrm{kHz}-500 \mathrm{kHz}]$ there is at least a pass-band channel of several $\mathrm{kHz}$ where the level of EMI does not interfere with the signals in the circuitry of Figure 20.

The change of $R_{B}$ can be implemented directly by the microcontroller in Figure 20 by using a digital-controlled potentiometer. The control of $R_{B}$ has been preferred to the control of $R_{A}$ since in (4) $R_{B}$ has a weight double of the one of $R_{A}$.

\section{Capacitive Detection}

9.1. Multidielectric Capacitive Sensing for Marble Industry. Once demonstrated in Section 8 the effectiveness of the sensing and reading principle some modifications have been applied to the schemes in Figures 18, 19, and 20 for a successful integration in a real marble machine. Indeed, as discussed in Section 2 and illustrated in Figure 24, in a marble machine the stone samples are placed on a plastic belt with a thickness $B$ less than $1 \mathrm{~cm}(0.5 \mathrm{~cm}$ typical $)$ sliding 


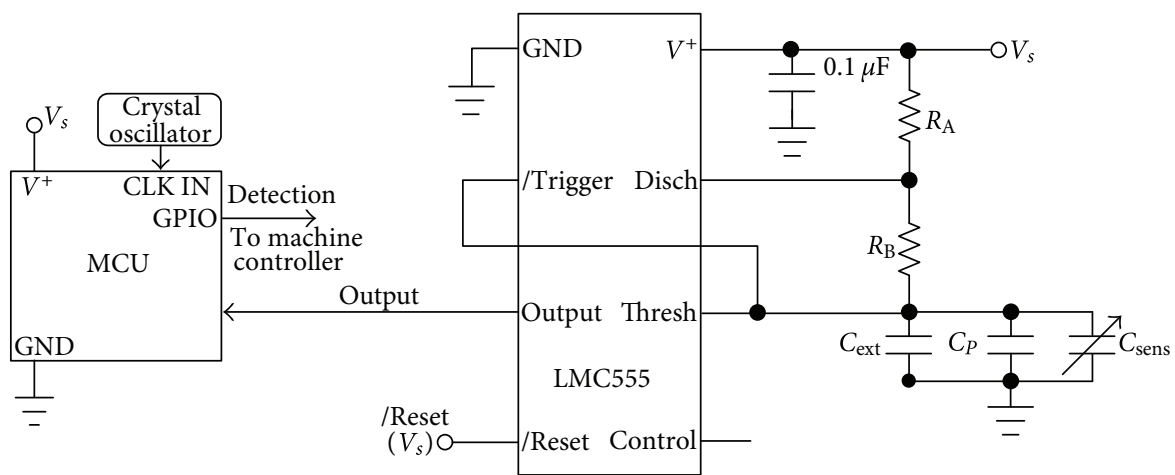

FIGURE 20: Front-end circuitry for capacitance change detection.

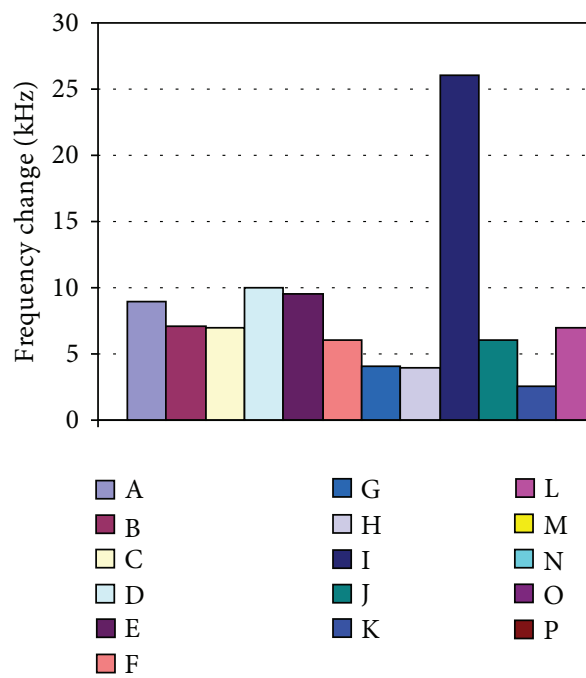

FIGURE 21: Frequency change for different stone samples, 2-plate capacitor.

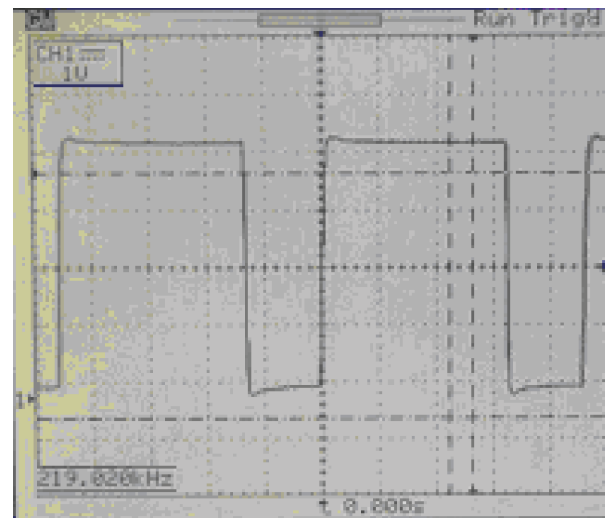

FIGURE 22: Snapshot of the astable output signal at $F_{0}$.

on a metallic plane. Hence the capacitive sensor can be simply realized suspending a conductive plate (top plate of the capacitor) over the already existing metallic plane of the industrial machine (bottom plate of the capacitor) at a distance $D$. Since the metallic plane surface is larger than

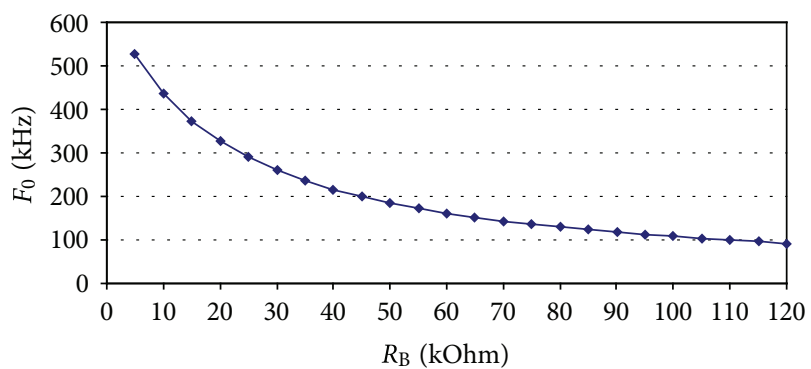

FIgURE 23: Change in $F_{0}$ versus change in $R_{B}$ for the circuit in Figure 20.

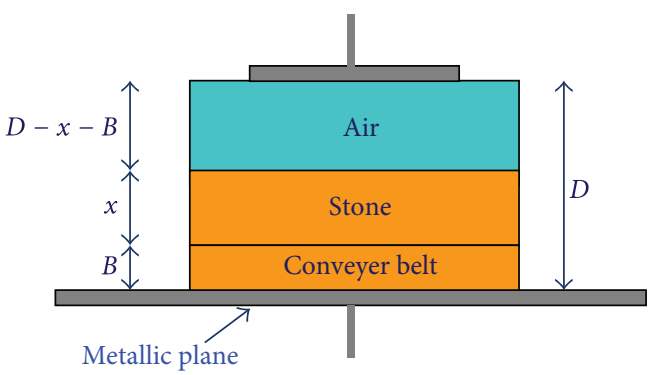

FIGURE 24: Multidielectric layer (air/stone/plastic belt) capacitive sensor inside the marble machine.

the metallic plate one, the method of image charges [33] can be applied: the new capacitor is equivalent to that of Section $8,(1)$ to $(3)$, but with an effective distance $2 D$. With respect to the analysis in Section 8 we have also to consider that in absence of the stone sample the filling dielectric is made up of a layer of $B=0.5 \mathrm{~cm}$ of plastic material (with $\varepsilon_{r b}$ between 2 and 3 ) and a layer of air of thickness $D-B$. The reference capacitance $C_{0}$ is now determined by

$$
C_{0}^{-1}=\left[\varepsilon_{o} \cdot \frac{S}{2(D-B)}\right]^{-1}+\left[\varepsilon_{r b} \varepsilon_{o} \frac{S}{2 B}\right]^{-1} .
$$

Considering $D=4.5 \mathrm{~cm}$ and $S=80 \mathrm{~cm}^{2}, C_{0}$ in (5) amounts to roughly $1 \mathrm{pF}$. When a stone sample of constant $\varepsilon_{r x}$ and thickness $x$ is passing through the metallic plates, a change in the capacitance value occurs since the capacitor is 


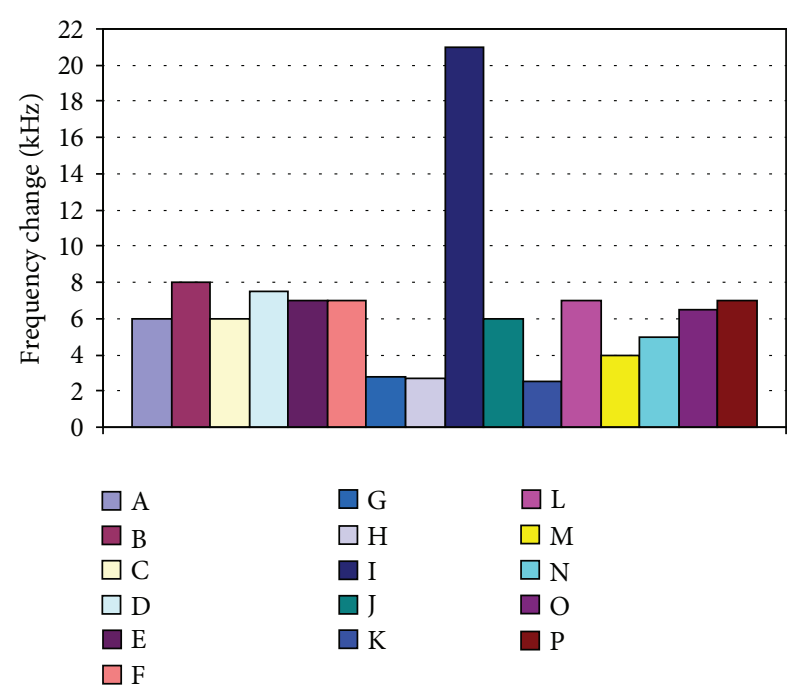

FIGURE 25: Frequency change for different stone samples, 1 plate capacitor.

filled by three dielectric layers: a layer of $B=0.5 \mathrm{~cm}$ due to the plastic belt, a layer of $x \mathrm{~cm}$ due to the stone, and a layer of air of size $D-x-B$. As a result of the stone interposition there will be a change of the capacitance $C_{\text {sens }}$ (the value increases) that can be read as a frequency change in the circuit of Figure 20 (the oscillation frequency decreases with respect to the reference value $F_{0}$ obtained when $C_{\text {sens }}=C_{0}$ ).

Implementing a test campaign with the new designed sensing circuit using the 16 stone samples mentioned in Section 3, different in size and shapes, and representative of the possible materials processed in marble industry (e.g., onyx, marble, granite,...etc.) the results of Figure 25 are obtained: the frequency change ranges from a minimum of $2.5 \mathrm{kHz}(\mathrm{K}$ sample in Figure 25) to a maximum of $21 \mathrm{kHz}$ (I sample in Figure 25) allowing a reliable detection of the stone samples.

The tests have been implemented also in presence of mud, marble dust, and powder obtaining similar results. As discussed in Section 8, since our goal is an on/off detection, a frequency change threshold of about $1 \mathrm{kHz}$ allows for sufficient margins against false or missing detections due to parasitic capacitance change, deposition of marble dust or mud, and changing of temperature conditions during the typical measure time (1 second and up to tens of seconds). Reference frequency variations in a larger time scale can be managed through the microcontrollers via software by monitoring $F_{0}$ and adapting the thresholds accordingly.

The correct behavior of the capacitive detection system with capacitive to frequency conversion has been confirmed also considering the presence of water inside the machinery.

In this case, see Figure 26, when the stone sample is not passing the reference capacitor value $C_{0}$ can be calculated considering a layer of size $B$ of roughly $0.5 \mathrm{~cm}$ due to the plastic belt in series with a layer of size $V$ (few $\mathrm{cm}$ ) filled by water and a layer of size $D-(V+B)$ filled by air. When the marble stone is interposed between the metallic plates it removes the water and the new capacitor value $C_{\text {sens }}$ can be

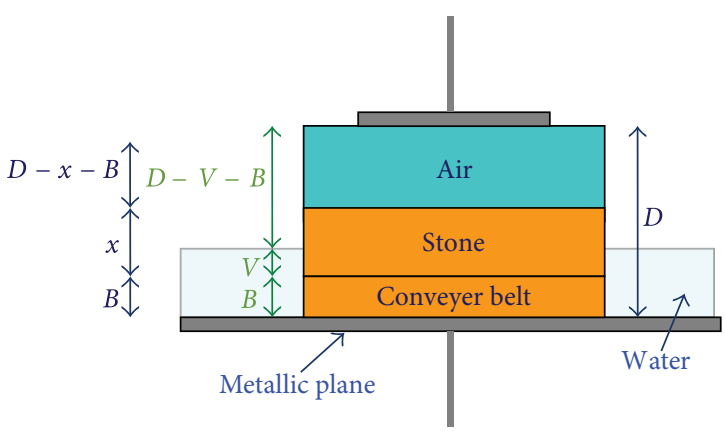

FIGURE 26: Multidielectric layer (air/stone/plastic belt) capacitive sensor inside the marble machine with water.

calculated considering a layer of size $B$ for the conveyer belt in series with a layer of size $x$ and dielectric constant $\varepsilon_{r x}$ and a layer of size $D-(x+B)$ filled by air. By detecting the capacitor change through a frequency change reusing the astable circuit (see Figure 20) the presence of a stone sample can be revealed in a reliable way. Repeating the test campaign using the 16 stone samples, different in size and shape, and in presence of water the frequency change is at minimum $1.5 \mathrm{kHz}$.

Summarizing, the proposed scheme can be easily implemented in a real marble machine, it is a low-complexity scheme with low maintenance cost, it is contactless, and it allows the reliable detection of stone samples also in presence of water, mud, and marble dust with enough margins against miss detections or false hits. The detection signal generated by the microcontroller may be sent to the main PLC controlling the working heads (see Figure 20). Though presenting a lower value of $C_{0}$ for a given surface $S$ of the plate, the single-plate capacitor solution has the great advantage versus the 2-plate capacitor discussed in Section 8 of not requiring the positioning of the second metallic plate under the conveyer belt, since the already existing metallic plane of the industrial machine is used. This way cabling and system maintenance are simplified.

9.2. Surface Capacitive Sensing for Marble Industry. In this section we propose a new capacitive sensor configuration, derived as an adaptation of the theory developed by Bozzi and Bramanti in [9]. Then we compare the obtained solution with the one proposed in Section 9.1.

In [9] Bozzi and Bramanti realized a capacitive sensor, see Figure 27, through the use of three copper conductive lines printed over a piece of FR4 dielectric material $\left(\varepsilon_{r} \sim=4.5\right)$ with a thickness $T$ of $10 \mathrm{~mm}$ : the central copper line, acting as signal line, is separated by a narrow gap $W$ from the two other lines, joined together at one end and acting as ground lines. The lines are separated by a distance $W=1 \mathrm{~mm}$, and therefore $W \ll T$.

As proved in [9] this device is sensitive to the average value of the dielectric permittivity ( $\varepsilon_{r 2}$ in Figure 27 ), of the material which is present at a distance $H$ from its surface, with $H \leq W$. As an example if the device in Figure 27 is put in contact with a material with dielectric value $\varepsilon_{r 2}$, the device has a capacitance expressed by (6) where $k$ is a constant whose 


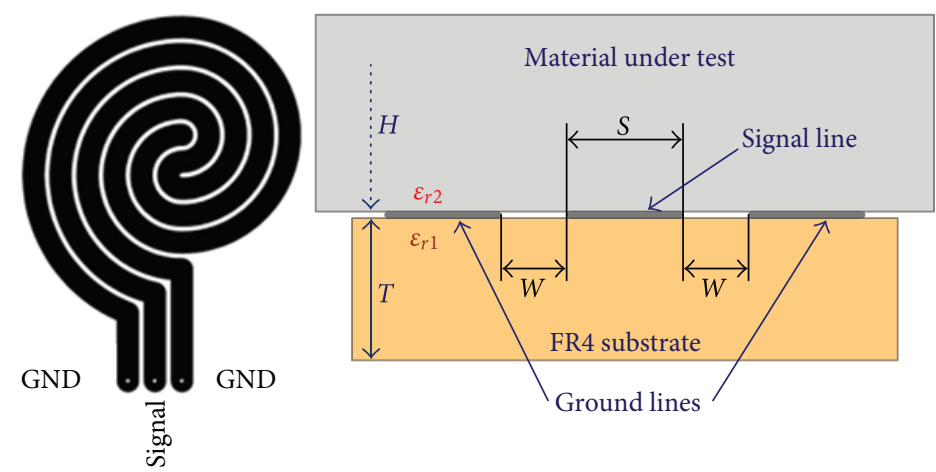

Figure 27: Surface capacitive sensor from [9].

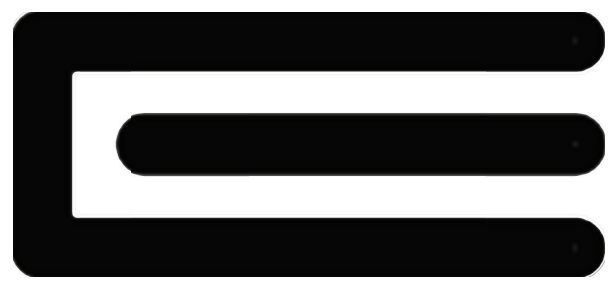

FIGURE 28: Modified surface capacitive sensor.

size depends on the ratio between the width of the lines, $S$, and their distance, $W$.

As suggested in [9] and realized in Figure 27, $S$ should be comparable to $W$ while the total track length $L$ should be much higher than $S$ and $W$ :

$$
C=2\left(\varepsilon_{r \mathrm{FR} 4}+\varepsilon_{r 2}\right) \cdot \varepsilon_{o} \cdot k .
$$

The above sensor has been used in [9] to reveal, on static samples, the surface value of the dielectric constant of materials by reading through a capacimeter the value of the capacitance between the signal and the ground lines. The sensor devised by Bozzi and Bramanti is a contact sensor for surface analysis: it is sensitive to the material property within a distance $H$ of about $1 \mathrm{~mm}$ from its surface.

Starting from the Bozzi and Bramanti theory we modified the sensor in order to

(a) use an FR4 plane with a thickness of $1.6 \mathrm{~mm}$, compliant with standard printed circuit board (PCB) technology and much lower than the $10 \mathrm{~mm}$ used in [9]; indeed the size used in [8] is not compliant with standard PCB technologies and hence implies a high device cost in case of industrial production;

(b) change the shape and size, see our new device in Figure 28, of the printed capacitor to be sensitive within a range $H$ of at least $2 \mathrm{~cm}$ from the surface.

The condition in the above point (b) is essential to allow a contactless detection of the presence of a stone sample. However, for the device in Figure 28, as in [9], the thickness of the sensitive area $H$ is determined by the distance $W$ between the conductive lines printed over the FR4 substrate.
To achieve a thickness $H$ of roughly $2 \mathrm{~cm}$ the device in Figure 28 has to be sized with a distance between the lines $W$ of about $2 \mathrm{~cm}$. The width $S$ of the printed conductive lines in Figure 28 is $2.5 \mathrm{~cm}$, while the larger side of the sensor has a size $L=$ $22 \mathrm{~cm}$ (according to the approach of Bozzi and Bramanti in [9] $L$ should be much higher than $W$ ). As a result, to achieve a thickness $H$ of roughly $2 \mathrm{~cm}$ a total area of $220 \mathrm{~cm}^{2}$ is required for the surface capacitive sensor, 2.75 times larger than the area of the capacitive sensors in Sections 8 and 9.1 which is $80 \mathrm{~cm}^{2}$. This is a bottleneck of the surface capacitive sensor presented in this section when compared to the capacitive sensor in Section 9.1. Indeed scaling all dimensions of Figure 28 to achieve a sensing thickness of $4 \mathrm{~cm}$, as in Section 9.1, the required area will increase up to $1300 \mathrm{~cm}^{2}$. For this reason the device in Figure 28 has been sized for a value of $H=2 \mathrm{~cm}$, a good tradeoff between sensitive thickness and sensor area.

Figure 29 reports the impedance $Z_{\text {in }}$, real and imaginary (absolute value) parts, offered by the sensor in Figure 28 as a function of the frequency, derived from an analysis with the electromagnetic simulator ADS (advanced design system) by Agilent Technologies. For sake of clarity two figures are reported referring to a frequency range from $10 \mathrm{~Hz}$ to $1 \mathrm{kHz}$ and a frequency range from $1 \mathrm{kHz}$ to $1 \mathrm{MHz}$.

The proposed sensor in Figure 28 can be suspended over the conveyer belt at a height of $3 \mathrm{~cm}$ (to leave enough space for the stone samples in the considered application case study of a thickness ranging from 1 to $3 \mathrm{~cm}$ ). When there is not any stone to detect $\varepsilon_{r 2}$ in (6) is 1 (air) and the sensed capacitive value represents the reference value $C_{0}$. When a stone is passing, the device can reveal its presence as a change in the capacitance value that can be converted through the astable circuit of Figure 20 in a frequency change. As already discussed when the stone is detected the capacitance value from (6) is increased and the oscillation frequency from (4) is decreased.

Implementing a test campaign with the sensing circuit designed in Figure 28 using the 16 stone samples, different in size and shape, already adopted in Sections 8 and 9.1, the results of Figure 30 are obtained.

The frequency changes in Figure 30 range from a minimum of $2 \mathrm{kHz}$ to a maximum of $7 \mathrm{kHz}$ allowing a reliable detection of the stone samples. 
TABLE 2: Main performance of the RFID systems (passive tags) for marble slab detection.

\begin{tabular}{|c|c|c|c|}
\hline RFID System & LF $125 \mathrm{kHz}$ & $\mathrm{HF} 13.56 \mathrm{MHz}$ & UHF $868 \mathrm{MHz}$ \\
\hline Radiated power & $100 \mathrm{~mW}$ & $<50 \mathrm{~mW}$ & $<500 \mathrm{~mW}$ \\
\hline Max. stone detection distance & $15 \mathrm{~cm}$ & $<8 \mathrm{~cm}$ & $<40 \mathrm{~cm}$ \\
\hline Best tag placement & \multicolumn{2}{|c|}{ Side surface of the marble slab } & Embedded in the conveyer belt or under it \\
\hline With water & \multicolumn{2}{|c|}{ Works } & Does not work \\
\hline With dust/dirt/stone residuals & \multicolumn{3}{|c|}{ Works } \\
\hline
\end{tabular}

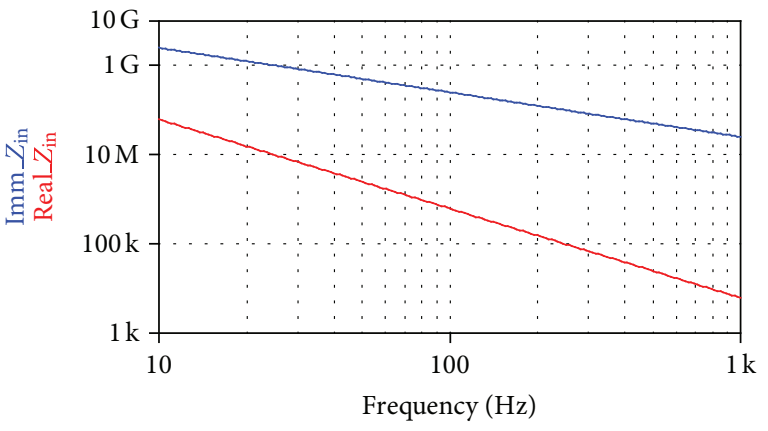

(a)

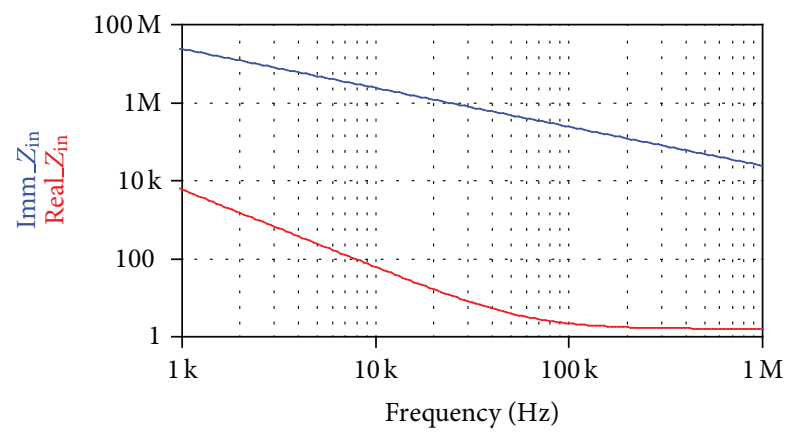

(b)

Figure 29: Real and imaginary (absolute value) parts of the surface capacitive sensor in Figure $28(10 \mathrm{~Hz}-1 \mathrm{kHz}$ and $1 \mathrm{kHz}-1 \mathrm{MHz}$ ranges) versus frequency.

Repeating the test in presence of water, marble dust, or mud we obtained similar results.

\section{Discussion on the Application of the Analyzed Sensing Technologies}

10.1. Comparison among the Different Analyzed Contactless Sensing Solutions. As illustrated in Section 4 to Section 7, the experimental analysis of the 4 RFID technologies has been carried out using a test setup reproducing environmental conditions representative of those found inside the marble machine, with water, mud, marble dust, and considering more than 16 different types of stones. The experimental results are schematically reported in Table 2.

Test results proved the following.

(i) Passive tags have to be preferred to active tags since the marble slab detection can be implemented with lower costs and easier system maintenance (e.g., no

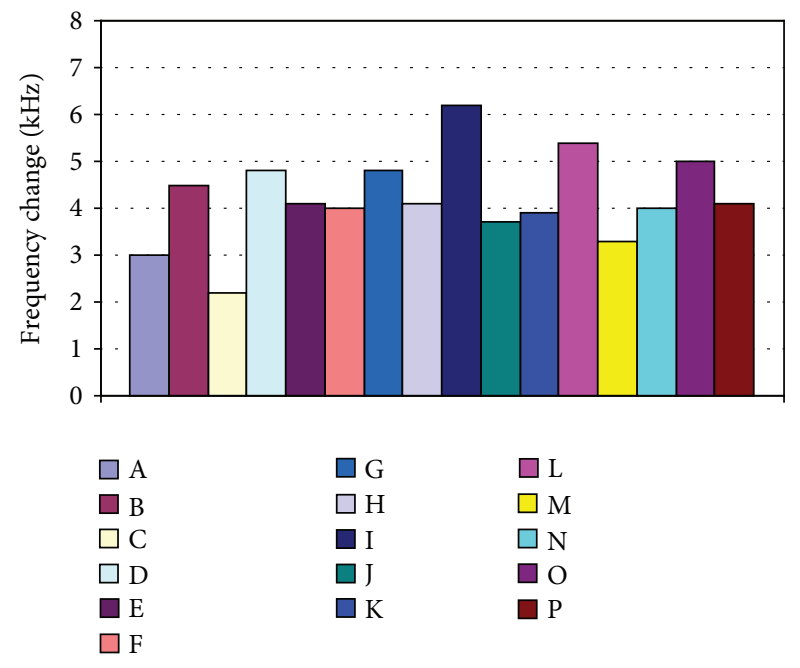

FIGURE 30: Frequency change versus stone sample, surface capacitor.

battery to be replaced). Table 2 summarizes the main performances of the analyzed RF detection systems using passive tags.

(ii) All types of stones are transparent to the tested $125 \mathrm{kHz} \mathrm{LF}$ and $13.56 \mathrm{MHz}$ HF radiations; hence for these RFID systems the most suitable strategy for a reliable detection is applying the tag on the side surface of each marble slab by means of a fast dry resin. This allows the detection, even in presence of water and dirt, of the tag and of the corresponding marble slab when the tag is passing in the area covered by the reader antenna. This strategy is useful anyway to confirm to the heads controlling system the presence of the marble slab in the expected point of the machine. The tag, placed in this position, can be used also to store information for logistic applications and for the traceability of the different industrial processes applied to each slab [17].

(iii) UHF communication at $868 \mathrm{MHz}$ and $2.45 \mathrm{GHz}$ is shielded by water and hence UHF systems can be used just outside the machine (dry environment): with respect to mechanical sensors, the RFID systems benefit of being contactless. With respect to optical systems the RFID ones are more robust to the presence of marble dust and dirt. The detection of the marble slab is possible according to the on/off strategy described in Section 6 by properly configuring the UHF system 
and possibly to the detection strategy presented in Section 7 concerning active RFIDs at $2.45 \mathrm{GHz}$. In the latter case, anyway, the behavior of the RFID system depends on the specific type of stone, and therefore the described detecting technique could be suitable just for applications involving a stone type a priori known.

It is worth noting that the above-described detecting techniques using passive tags require low-cost tags (one for each marble slab or a set embedded in the conveyer belt) and an antenna for each head of the machine or serving a few consecutive heads. Multiple antennas (e.g., up to 4 for the RFID system in [24]) can be controlled by the same reader positioned outside the machinery since the reader and its antenna are typically connected through cables whose length amounts to some meters.

The application of contactless sensing of the presence and/or the position of the marble slab by means of RFID technologies also have a direct benefit on the possibility to avoid or reduce cabling inside or outside the machine for sensor data transmission to the machine controlling system. For passive RFID tags, no cabling among them is needed, while in the case of active RFID tags (usable only outside the machine) supply should be provided to tags (batteries need maintenance, and energy harvesting appears not to be practical or possible at the required transmission rate).

Passive RFID tags implement a wireless sensor network coordinated by the RFID reader.

The possibility offered by RFID technologies of multitag detection and collision management system allows for the implementation of linear sensor (tag) arrays placed under the conveyer belt at the machine entrance stage. For a typical case study, a tag spacing of $10 \mathrm{~cm}$ would allow a usable spatial resolution in slab sampling. At this aim, about 30 tags can constitute a tag array covering the conveyer belt width (in the order of $3 \mathrm{~m}$ ), and, depending on the particular RFID technology used, multitag reading is possible at a rate sufficient to read a tag array every one-two seconds by means of one or more RFID antennas and/or readers. In such a way, slab presence detection and slab shape detection may be realized for process control. The tag IDs are provided as data output by the RFID readers on a serial or Ethernet port. RFID readers may be connected through a point-to-point (or multipoint-to-point) connection to

(i) the PLC, if its processing power is sufficient to command/configure the RFID readers, receive detected tag IDs, and transform them into slab sampling information;

(ii) an intermediate intelligent data collecting and processing device, for example, a microcontroller-based one, able to command/configure the reader and to apply detection algorithms, sending to the PLC only the final detection data.

Concerning the LF and HF RFID systems, tags are only one or a few for each slab, the reader and its antenna are mounted on the moving spindle bridge, and tag ID data may be transmitted to the PLC wirelessly or by means of a wired bus. In the latter case there are some additional issues due to the movement of the spindle bridge.

Concerning the two capacitive sensing solutions we proposed in Sections 9.1 and 9.2, the experimental campaign carried out with real working conditions highlighted the main advantages and disadvantages of each approach.

As already discussed in Section 9.2, if the two sensors are realized with a comparable area occupation then the PCB-printed surface capacitor offers a reduced detection distance. Obviously, to achieve a similar detection distance the surface capacitor requires a larger area. Indeed for the marble machine application case study a total area of $220 \mathrm{~cm}^{2}$ is required for the surface capacitive sensor in Section 9.2, 2.75 times larger than the area of the sensor in Section 9.1 which is $80 \mathrm{~cm}^{2}$.

As far as the detection robustness is concerned it is clear that, in average, the frequency changes in Figure 30 for the surface capacitor are lower than those in Figure 25 for the single-plate capacitor suspended over the machine metallic plane. However the minimum detected frequency change value in both solutions is comparable: $2.5 \mathrm{kHz}$ in Figures 25 and $2 \mathrm{kHz}$ in Figure 30. Since our goal is an on/off detection of the stone samples, as previously discussed a threshold of about $1 \mathrm{kHz}$ is adequate for both capacitive sensor systems in Sections 9.1 and 9.2. This allows, for both systems, sufficient margins against false or missing detections due to parasitic capacitance change, deposition of marble dust or mud, and change of temperature conditions.

The main advantages of the capacitor described in Section 9.2 versus the solution in Section 9.1 are

(i) the possibility to avoid cabling between the suspended plate and the metallic base of the marble machine;

(ii) the possibility to implement on the same PCB the capacitive sensor, the front-end measurement circuitry, and the interface for data communication (wired or wireless).

With the surface sensing approach, cabling and connection problems are reduced and system maintenance is simplified.

10.2. Case Study Application of Networked Sensing Data Collection in Marble Machines. In this section we discuss the application of the detection sensors outside and inside the machine and the concerning data generation. As application case study, we consider the surface capacitive sensors, as the collection of their output data is performed by means of an architecture resulting in an extension of the one used for the UHF RFID detection and data collection technologies applied at the machine entrance stage.

The arrangement of several sensors into a linear array implements a multipoint sensing scheme for the spatial sampling of the slab, as currently done by mechanical or optical sensors just at the entrance stage of the machine.

The linear array is mounted perpendicularly to the movement direction of the conveyer belt and spans for its entire width, see Figure 31 . We indicate with $w_{\mathrm{cb}}$ the width of the 


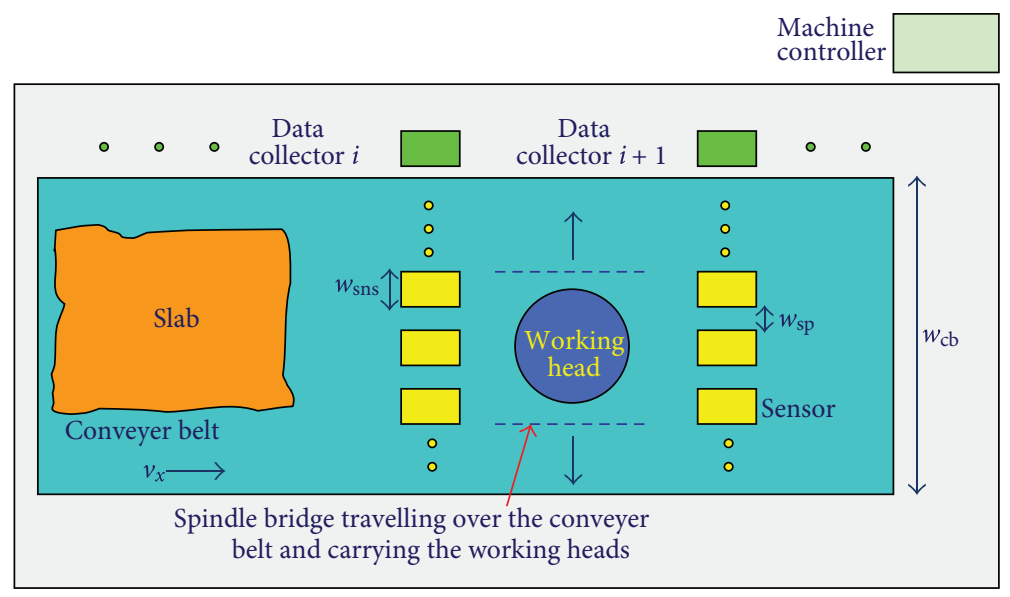

FIGURE 31: Schematic representation of the machine sensing and data collection architecture.

conveyer belt, with $w_{\text {sn }}$ the width of each sensor, and with $w_{\text {sp }}$ the spacing among two sensors in the array.

We have therefore a number of sensors $n_{\text {sns }}$ given by

$$
n_{\mathrm{sns}}=\text { floor }\left(\frac{w_{\mathrm{cb}}}{\left(w_{\mathrm{sn}}+w_{\mathrm{sp}}\right)}\right) \text {. }
$$

Sensor output data have to be collected, processed, and transmitted to the machine controller, which, in case of the sensors mounted inside the machine, compares sensor data with data previously drawn from the sensors at the entrance stage, being therefore able to be aware of unexpected slab displacements.

As already mentioned in the beginning of the present Section 10.2, as a case study we consider an array of capacitive sensors usable also inside the machine.

Each capacitive sensor and the relevant front-end circuitry for oscillation generation and frequency detection implement a node of the sensing array, connected to the other sensing nodes through a wired bus (e.g., RS-485, I² C, CAN, etc.).

For the implemented surface capacitive sensors of Figure 28 , whose lower side is $10 \mathrm{~cm}$ long, we consider the following data:

$$
\begin{aligned}
& w_{\text {sn }}=10 \mathrm{~cm}, \text { width of the surface capacitor PCB; } \\
& w_{\mathrm{sp}}=10 \mathrm{~cm} \text {, spacing among capacitive sensors; } \\
& w_{\mathrm{cb}}=3.0 \mathrm{~m} \text {, width of the conveyer belt. }
\end{aligned}
$$

We have therefore a number of sensors given by (7) which leads to $n_{\text {sns }}=15$.

As illustrated in the schematic representation in Figure 20, we propose a surface capacitive sensor connected to a small supplementary PCB (that can be further integrated with the sensor on the same PCB) integrating an oscillator (555 timer), a low-cost 8-bit microcontroller (Atmel ATmega 16 , which has been chosen for demonstration purposes and is largely sufficient in terms of the features needed), and a communication interface for multidrop bus communication that in the application case study was chosen as the $\mathrm{I}^{2} \mathrm{C}$ bus directly driven by the microcontroller in slave mode. Of course, such a communication bus may be substituted by other industrial data buses in an on-field application. The data collector (see Figure 31) is a microcontroller-based (Atmel ATmega 16) device acting as $\mathrm{I}^{2} \mathrm{C}$ master, reading frequency data from the different sensors and implementing proper slab detection and slab shape sampling algorithms whose results are transmitted to the machine controller.

More specifically, the microcontroller on each capacitor enables/disables the oscillating circuit, measures the capacitor oscillation frequency, and sends it every regular interval to the master microcontroller.

The slab detection and slab shape sampling strategies are devolved to the master microcontroller and not to the onboard intelligence of each sensor, as the master microcontroller is aware of the oscillation frequencies of all the sensors in the array, and can therefore

(i) detect quiescent oscillation frequency during a learning/calibration phase performed periodically (without any slab passing) or at machine start and set different detection threshold levels for frequencies of the different sensors; moreover, quiescent frequency may be quite different from sensor to sensor due to the spread in physical and electrical characteristics of its components and to the surrounding environment, for example, proximity to metallic parts of the machine, and so forth;

(ii) detect, during operations, frequency derating due to variations in the water level, in the environment temperature, and so forth, and change detection threshold accordingly.

The master microcontroller (data collector) communicates with the main machine controller wirelessly or through a wired data bus, sharing the communication channel with the other master microcontrollers managing other sensor arrays, and transmits to the machine controller detection data. Each data collector also receives commands from the machine controller, for example, to activate/deactivate sensor arrays, and so forth. The above architecture, in which 
single sensor arrays and the respective data collector devices are connected to the same cables (supply and, for wired communication, to the data bus), allows for reduced cabling: in the mechanical sensors at the machine entrance stage, see Figure 3, tens of cables, one for each sensor tip, are connected to the PLC. The communication between each sensor and the array data collector occurs with a relatively low rate. In the application case study, the oscillation frequency is coded using 2 bytes $(8 \mathrm{~Hz}$ resolution for a full scale frequency of $524288 \mathrm{~Hz}$ ); the number of sensors for each array is $n_{\mathrm{sns}}=15$, and each sensor is assigned a 1-byte $\mathrm{I}^{2} \mathrm{C}$ address; sensor data is transmitted every $T=1 \mathrm{~s}$; the slab is transported on the conveyer belt at a $v_{x}=5 \mathrm{~cm} / \mathrm{s}$. In such a case, the obtained spatial sampling is $s_{x}=v_{x} \cdot T=5 \mathrm{~cm}$ and $s_{y}=w_{\mathrm{sn}}+w_{\mathrm{sp}}=$ $20 \mathrm{~cm}$.

If no transmission data integrity check or redundancy is used, the needed transmission rate is about $f_{\mathrm{TX}}=n_{\mathrm{sns}} \cdot 3 / T=$ $45 \mathrm{~B} / \mathrm{s}$, that is, well under $1 \mathrm{kbit} / \mathrm{s}$, a communication speed reachable on commonly used data buses, even in an industrial scenario.

Of course, in an industrial, electrically noisy environment, and due to the impact on the process safety itself, it is necessary to implement data integrity check and filtering schemes, at the sensor raw data output level (a checksum byte for frequency data) and at processing level by the data collector (e.g., removing isolated detection/nondetection points near frequency threshold).

Concerning the implementation costs, a capacitive-based sensing solution could in general be less expensive than a RFID-based sensing one. As an example, a cost estimation for a RFID-based linear array sensor (UHF system, 4 antennas) leads to a cost higher than the corresponding capacitive linear array sensor. It should be anyway noticed that the price of machines for marble slab working is in the order of one to several hundreds of thousands of euros, and therefore the cost of electronics (estimation in the order of thousands of euros) would have a limited impact on the whole machine cost. Anyway, for a correct comparison of the implementation cost, also the impact on the mechanics of the machine sections involved (e.g., the metallic basement) should be evaluated by means of a detailed mechanical design review, that is beyond the scope of the present paper, but the impact appears reasonably limited in comparison to the overall machine cost. As marble machines can operate for several years, the overall cost saving due to the possibility to prevent or reduce damages to the conveyer belt and the consequent machine stops also contributes to justify the extra cost needed for the sensing application. Moreover, the RFID technology might be used not only for slab detection and process control but also to manage the marble logistics during the entire travel and stocking in the working facility, as an example in [34].

\section{Conclusion}

The paper has presented an experimental analysis towards the application of RFID and capacitive wireless sensing technologies to process control in the marble industry. The final aim was the detection of the presence of a marble slab under the abrasive/cutting head inside an industrial machine or outside at the machine entrance stage. The proposed techniques try to overcome issues related to currently used detection sensors: mechanical or optical devices which suffer from deterioration and dirt-related problems and do not provide the main machine controlling system with feedback signals about the correct alignment between each marble slab and the corresponding cutting/abrasive head inside the machine. This may result in safety issues, costly damages for the machine itself, and a long production stop.

The experimental results for the analyzed RFID technologies using a test setup reproducing the marble machine environment (with water, mud, marble dust) and considering more than 16 different types of stones have led to depict advantages and limits for each tested technology and to define their possibility of use at the entrance stage and/or inside the machine.

The proposed capacitive sensing solutions are optimized for the contactless detection of stone samples inside the industrial machine. Compared to the state-of-the-art detection systems, the proposed solutions allow for a reliable detection while being of low complexity and with low maintenance cost, can be easily integrated with the machine controlling system and are robust to harsh environment conditions. We have then proposed an application case study for surface capacitive sensors representative of a sensing and data collecting network architecture for slab detection outside and inside the machine, providing the machine controlling system with information about the slab position during working. Such architecture is suitable to a more general application, not limited to cutting/polishing processes in marble industry, exploiting the proposed contactless sensing techniques applied in the automation of industrial machinery.

\section{Acknowledgments}

This work has been supported by the Tuscany Region DOCUP program in collaboration with Celver Elettronica srl. Discussions with R. Massini, A. Carrafiello, and E. Valentini are gratefully acknowledged.

\section{References}

[1] Barsanti Spa, "Gangsaw TLD 60A/80A machine," 2009, http:// www.barsantimacchine.it.

[2] Barsanti Spa, "LCA200 marble polishing machine," 2008, http://www.barsantimacchine.it.

[3] D. Miorandi, E. Uhlemann, S. Vitturi et al., "Guest editorial: special section on wireless technologies in factory and industrial automation," IEEE Transactions on Industrial Informatics, vol. 3, no. 2, pp. 95-98, 2007.

[4] Celver srl, "Archimedes optical system," http://www.celver.it/ new-site/prodotti/prodotti-archimedes.asp.

[5] M. Bramanti et al., "A procedure to detect flaws inside large sized marble blocks by ultrasound," Subsurface Sensing Technologies and Applications, vol. 2, no. 1, pp. 1-13, 2001.

[6] M. A. Selver, O. Akay, E. Ardali, B. A. Yavuz, O. Önal, and G. Özden, "Cascaded and hierarchical neural networks for classifying surface images of marble slabs," IEEE Transactions on 
Systems, Man and Cybernetics Part C, vol. 39, no. 4, pp. 426-439, 2009.

[7] J. D. Luis-Delgado, J. Martínez-Alajarín, and L. M. TomásBalibrea, "Classification of marble surfaces using wavelets," Electronics Letters, vol. 39, no. 9, pp. 714-715, 2003.

[8] S. A. Coker and Y. C. Shin, "In-process control of surface roughness due to tool wear using a new ultrasonic system," International Journal of Machine Tools and Manufacture, vol. 36, no. 3, pp. 411-422, 1996.

[9] E. Bozzi and M. Bramanti, "A planar applicator for measuring surface dielectric constant of materials", IEEE Transactions on Instrumentation and Measurement, vol. 49, no. 4, pp. 773-775, 2000.

[10] D. Vaccaneo, L. Sambuelli, P. Marini, R. Tascone, and R. Orta, "Measurement system of complex permittivity of ornamental rocks in L frequency band," IEEE Transactions on Geoscience and Remote Sensing, vol. 42, no. 11, pp. 2490-2498, 2004.

[11] S. Saponara, F. Iacopetti, A. Carrafiello, E. Valentini, L. Fanucci, and B. Neri, "Capacitive sensors for process control in industrial marble machines," in Proceedings of the 5th IEEE International Workshop on Intelligent Data Acquisition and Advanced Computing Systems: Technology and Applications (IDAACS '09), pp. 142-147, Rende, Italy, September 2009.

[12] B. Osoincah, "Proximity capacitive sensor technology for touch sensing applications," Freescale White Paper, 2007.

[13] S. Saponara, F. Iacopetti, A. Carrafiello, L. Fanucci, B. Neri, and R. Massini, "Experimental analysis towards the application of RFID technologies in industrial marble machines," in Proceedings of the 5th IEEE International Workshop on Intelligent Data Acquisition and Advanced Computing Systems: Technology and Applications (IDAACS '09), pp. 67-71, Rende, Italy, September 2009.

[14] I. Ar and Y. S. Akgul, "A generic system for the classification of marble tiles using Gabor filters," in Proceedings of the 23rd International Symposium on Computer and Information Sciences (ISCIS '08), pp. 1-6, October 2008.

[15] J. Martínez-Alajarín, J. D. Luis-Delgado, and L. M. TomásBalibrea, "Automatic system for quality-based classification of marble textures," IEEE Transactions on Systems, Man and Cybernetics Part C, vol. 35, no. 4, pp. 488-497, 2005.

[16] K. Finkenzeller, RFID Handbook: Fundamentals and Applications in Contactless Smart Cards, Radio Frequency Identification and Near-Field Communication, John Wiley \& Sons, 3rd edition, 2010.

[17] RFIDline, "RFid project: special technology on marble and granite," 2007, http://www.rfidstone.com.

[18] R. Wessel, "Italian stone supplier uses RFID to track marble, granite," RFIDJournal, 2007.

[19] K. Kwon, J. Ryu, J. Sohn, and I. Chung, "Intelligent process control system with RFID cuboid," in Proceedings of the 11th International Conference on Electronic Commerce (ICEC '09), pp. 1-8, Taipei, Taiwan, 2009.

[20] G. Fenu and P. Garau, "RFID- based supply chain traceability system," in Proceedings of the 35th Annual Conference of the IEEE Industrial Electronics Society (IECON '09), pp. 2672-2677, November 2009.

[21] B. S. Choi, J. W. Lee, and J. J. Lee, "An improved localization system with RFID technology for a mobile robot," in Proceedings of the 34th Annual Conference of the IEEE Industrial Electronics Society (IECON '08), pp. 3409-3413, November 2008.

[22] EM Microelectronic, "EM4102 Read only contact less identification device," 2005.
[23] PSION Teklogic, "Workabout Pro guide," 2004.

[24] CAEN SpA, "A928 long range UHF reader data sheet," 2007, http://www.caen.it/rfid.

[25] Open 2.4 GHz RFID Sputnik, http://www.openbeacon.org.

[26] Y.-C. Choi, M.-W. Seo, Y.-H. Kim, and H.-J. Yoo, "A multi standard 13.56 MHz RFID reader system," in Proceedings of the International Technical Conference on Circuit/System, Computers and Communications (ITC-CSCC '08), pp. 1073-1076, 2008.

[27] N. Choi et al., "Design of a $13.56 \mathrm{MHz}$ RFID system," in Proceedings of the 14th International Conference on Advanced Communication Technology (ICACT '12), pp. 840-843, 2006.

[28] Beijing Hongchangtag Tech-Sci Development Inc, "HCTHFR-80507,” 2012, http://www.hongchangtag.com/product .asp? $\mathrm{id}=15 \& \mathrm{f} 1 \mathrm{id}=1 \& \mathrm{f} 2 \mathrm{id}=19 \& \mathrm{f} 1=3$.

[29] M. Chaplin, "Water structure and science; water and microwaves," http://www.lsbu.ac.uk/water.

[30] M. Kollar, "Measurement of capacitances based on a flip-flop sensor," Sensors \& Transducers Magazine, vol. 35, no. 8-9, pp. $1-7,2003$.

[31] G. Brasseur, "Design rules for robust capacitive sensors," IEEE Transactions on Instrumentation and Measurement, vol. 52, no. 4, pp. 1261-1265, 2003.

[32] L. Fanucci, S. Saponara, and A. Morello, "Power optimization of an 8051-compliant IP microcontroller," IEICE Transactions on Electronics, vol. 88, no. 4, pp. 597-600, 2005.

[33] D. Dugdale, Essentials of Electromagnetism, The Macmillan Press, London, UK, 1993.

[34] F. Marco, System and method for a plant for working natural stones. European Patent Application 10425405.7, 2010. 

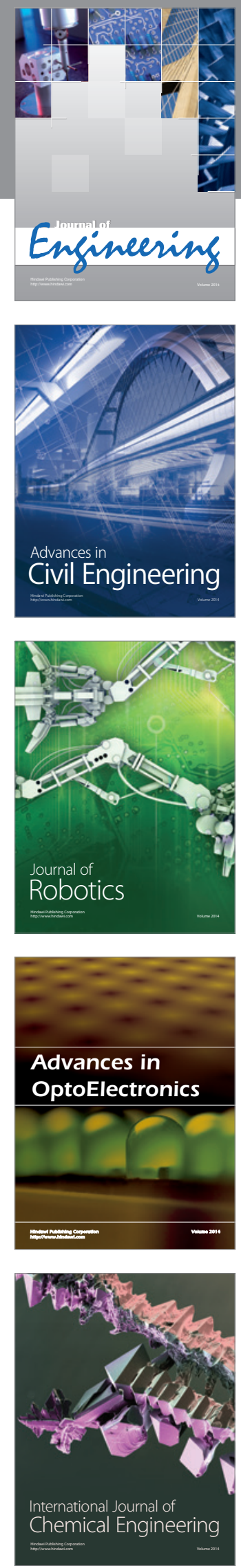

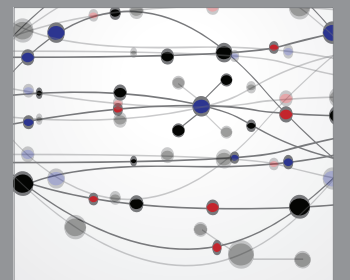

The Scientific World Journal
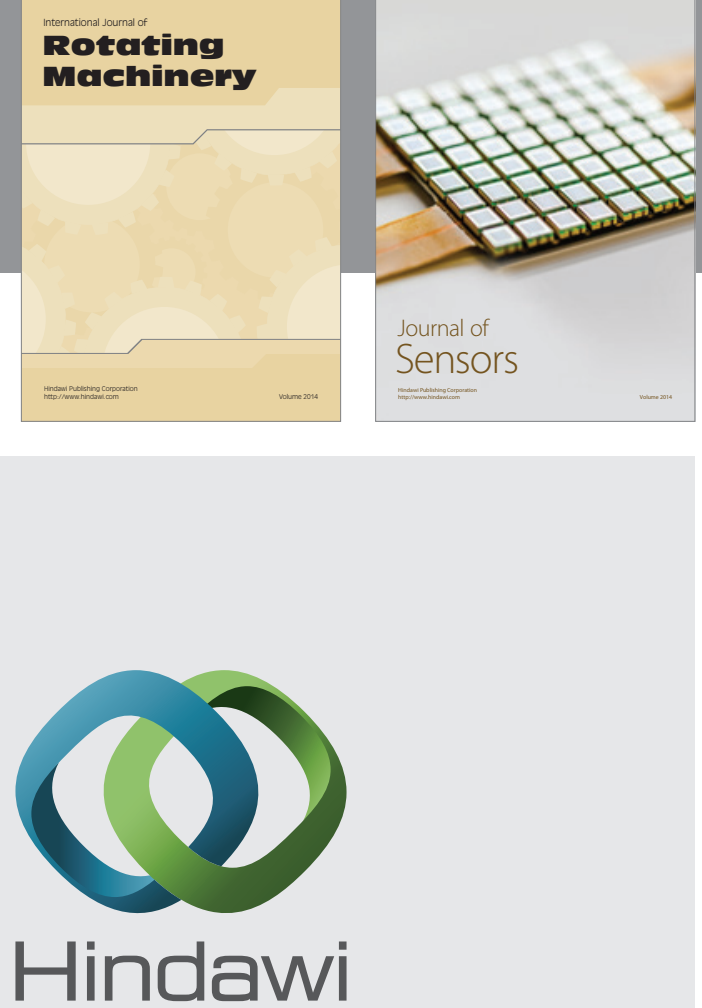

Submit your manuscripts at http://www.hindawi.com
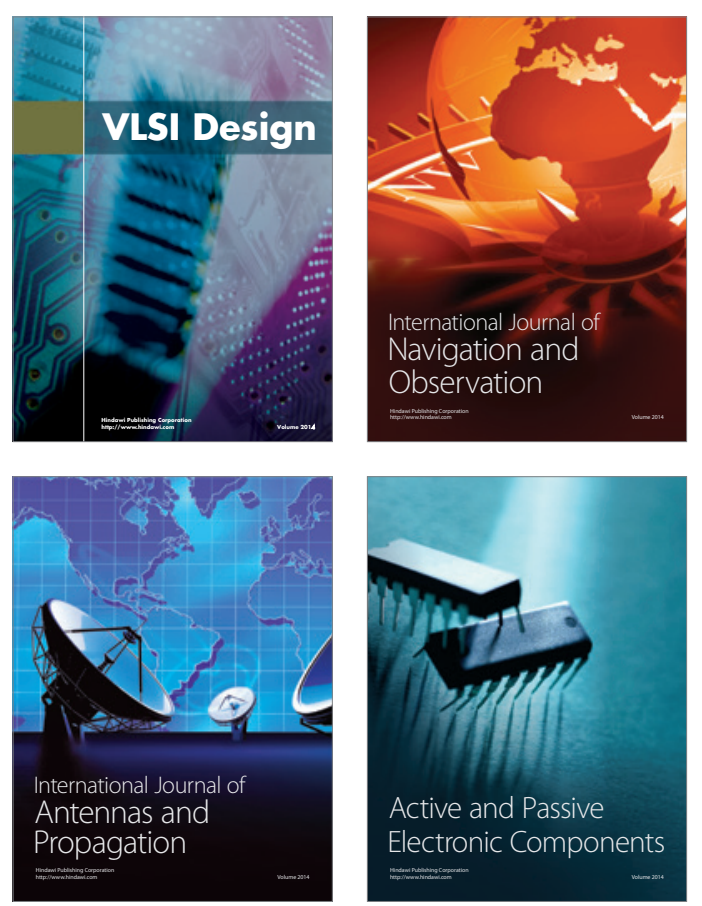
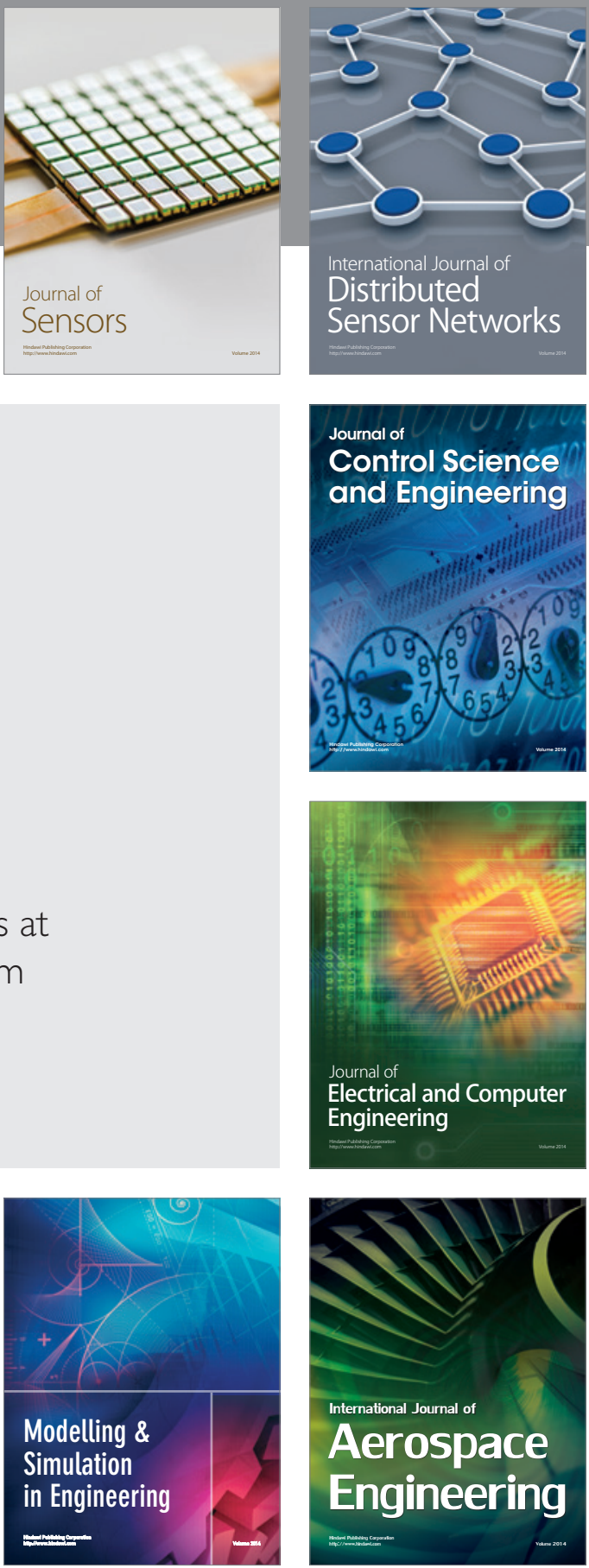

Journal of

Control Science

and Engineering
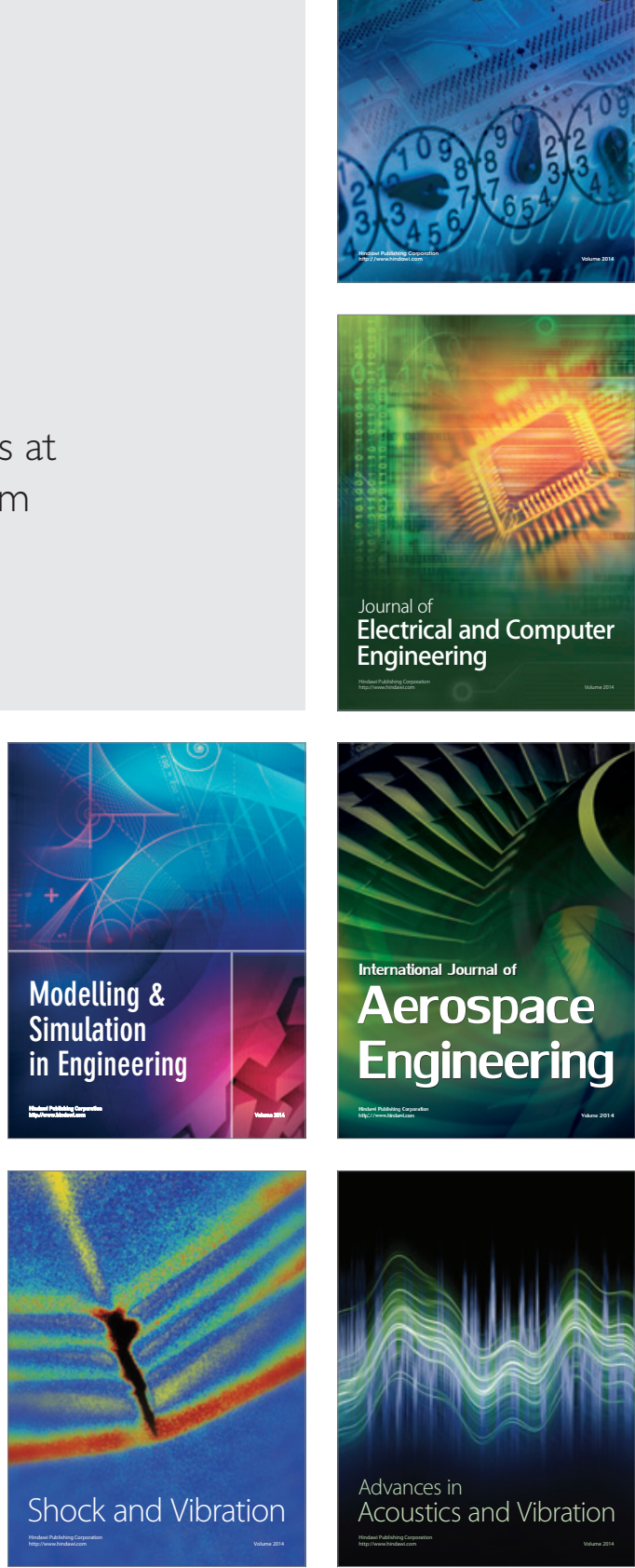